\title{
BMS flux algebra in celestial holography
}

\author{
Laura Donnay and Romain Ruzziconi \\ Institute for Theoretical Physics, TU Wien, \\ Wiedner Hauptstrasse 8-10/136, A-1040 Vienna, Austria \\ E-mail: Laura.donnay@tuwien.ac.at, romain.ruzziconi@tuwien.ac.at
}

ABSTRACT: Starting from gravity in asymptotically flat spacetime, the BMS momentum fluxes are constructed. These are non-local expressions of the solution space living on the celestial Riemann surface. They transform in the coadjoint representation of the extended BMS group and correspond to Virasoro primaries under the action of bulk superrotations. The relation between the BMS momentum fluxes and celestial CFT operators is then established: the supermomentum flux is related to the supertranslation operator and the super angular momentum flux is linked to the stress-energy tensor of the celestial CFT. The transformation under the action of asymptotic symmetries and the OPEs of the celestial CFT currents are deduced from the BMS flux algebra.

Keywords: AdS-CFT Correspondence, Classical Theories of Gravity, Space-Time Symmetries

ArXiv EPrint: 2108.11969 


\section{Contents}

1 Introduction 1

2 Asymptotically flat spacetimes 2

3 Conformal fields on the celestial Riemann surface $\quad 6$

4 Generators and momenta $\quad 8$

$5 \quad$ BMS flux algebra $\quad 12$

6 Momentum fluxes and CCFT operators 14

7 Constraints on CCFT $\quad 17$

8 Discussion 20

\section{Introduction}

Celestial holography aims at establishing a holographic description of quantum gravity in four-dimensional asymptotically flat spacetime in terms of a two-dimensional conformal field theory, called celestial CFT (or CCFT for short), living on the boundary celestial Riemann surface. This program exploits the richness of the asymptotic symmetry structure of the spacetime [1-6] to constrain the potential candidate for the celestial dual theory. Conformal symmetries of the CCFT are induced by superrotations which are part of the (extended) Bondi-Metzner-Sachs (BMS) asymptotic symmetries in the bulk theory [5, 710]. In celestial holography, each scattering particle in the bulk spacetime is associated to an operator that lives on the boundary celestial Riemann surface. In those terms, soft theorems in the bulk spacetime, corresponding to Ward identities of the gravitational $S$ matrix for the extended BMS symmetries, are implemented by $2 d$ currents in the CCFT; see [11] for a review. In particular, the supertranslation current has Ward identities that are equivalent to the leading soft graviton theorem [6, 12], while the sub-leading soft theorem is obtained by the insertion of a holographic stress-tensor [13]. A natural basis to describe massless asymptotic particles in celestial holography can be obtained by applying a Mellin transform with respect to the energy of the external particle, which maps energy eigenstates to boost eigenstates and hence makes the conformal properties more manifest [4, 14-17].

In [18], the coadjoint representation of the BMS group in four dimensions has been constructed. It acts on a set of conformal fields that have been identified with local expressions of the solution space of non-radiative asymptotically flat spacetimes at null infinity 
through a (pre-)momentum map. In presence of radiation, the transformation of the gravitational solution space becomes more complicated and the coadjoint representation of the BMS group is not sufficient to describe it. Furthermore, the BMS surface charges become non-integrable [19] and one needs additional inputs to select a meaningful integrable part. The algebra requires the use the modified Barnich-Troessaert bracket [20], leading to a field-dependent 2-cocycle (see also [21] for a detailed analysis from double-soft limits of amplitudes). As shown in [22, 23], the latter can be re-absorbed in the definition of the modified bracket by using the Noetherian split between integrable and non-integrable parts.

Instead of working with local expressions of the solution space at finite value of retarded time $u$, one could consider fluxes that correspond to integrated expressions over $u$. This point of view is closer to the spirit of celestial holography where the retarded time does not appear explicitly in the CCFT. It was shown in $[24,25]$ that, provided one chooses an appropriate integrable part in the BMS surface charges, the algebra of associated BMS fluxes closes under the standard bracket. The prescription that we consider here is based on $[25,26]$ and has the following important properties: (i) the flux algebra closes under the standard bracket, (ii) the fluxes vanish when evaluated on vacuum solutions (namely solutions with identically vanishing Riemann tensor that are constructed in [27-29]), (iii) the fluxes vanish for non-radiative spacetime solutions, (iv) the fluxes are finite provided one chooses the appropriate falloffs in $u$.

In this paper, we identify some non-local combinations of the solution space of fourdimensional asymptotically flat spacetimes that transform in the coadjoint representation of the extended BMS group in presence of radiation. We call these expressions the BMS momentum fluxes since they are involved in the BMS fluxes discussed in [25]. The inclusion of the superrotations in the analysis requires a meticulous treatment of the $2 d$ Liouville stress-tensor discussed in [26, 27]. In a second step, we propose a new prescription to split the fluxes into soft and hard parts, so that the associated soft and hard phase spaces factorize. We then relate the soft BMS momentum fluxes with the supertranslation operator and the stress-tensor of the CCFT. We provide the precise expressions of these CCFT currents in terms of the bulk metric and deduce their transformation laws under extended BMS transformations. Finally, from the BMS flux algebra, we deduce the OPEs of the BMS momentum fluxes and recover the OPEs of the CCFT operators.

\section{$2 \quad$ Asymptotically flat spacetimes}

In this section, we describe the bulk side of the celestial holographic description by reviewing the analysis of four-dimensional asymptotically flat spacetimes at null infinity, denoted $\mathscr{I}^{+}$, in Bondi gauge [1-3]. We mainly follow the notations and conventions of $[5,26]$. In Bondi coordinates $\left(u, r, x^{A}\right), x^{A}=(z, \bar{z})$, the spacetime metric reads as

$$
d s^{2}=e^{2 \beta} \frac{V}{r} d u^{2}-2 e^{2 \beta} d u d r+g_{A B}\left(d x^{A}-U^{A} d u\right)\left(d x^{B}-U^{B} d u\right),
$$

where $\beta, V, g_{A B}, U^{A}$ are functions of $\left(u, r, x^{A}\right)$ and the transverse metric $g_{A B}$ satisfies the determinant condition

$$
\partial_{r}\left[r^{-4} \operatorname{det}\left(g_{A B}\right)\right]=0 .
$$


We consider the asymptotically flat spacetimes satisfying the boundary conditions

$$
\beta=\mathcal{O}\left(r^{-1}\right), \quad \frac{V}{r}=-1+\mathcal{O}\left(r^{-1}\right), \quad U^{A}=\mathcal{O}\left(r^{-1}\right), \quad g_{A B}=r^{2} \stackrel{\circ}{q}_{A B}+r C_{A B}+\mathcal{O}\left(r^{-1}\right),
$$

where

$$
\stackrel{\circ}{q}_{A B} d x^{A} d x^{B}=2\left(\Omega_{S} \bar{\Omega}_{S}\right)^{-1} d z d \bar{z}, \quad \Omega_{S}=\frac{1+z \bar{z}}{\sqrt{2}}=\bar{\Omega}_{S}
$$

is unit sphere metric and $C_{A B}(u, x)$ is a 2-dimensional symmetric traceless tensor called the asymptotic shear. Let us make some comments on the choice of falloffs $(2.3),(2.4)$ :

- We allow for possible puncture singular violations of the above boundary conditions to accommodate with the Witt $\oplus \overline{\text { Witt }}$ superrotations symmetries $[5,7,8]$ that we discuss below. In particular, we consider the topology of the 2-punctured sphere as celestial Riemann surface $\mathcal{S} \simeq \mathscr{I}^{+} / \mathbb{R}[18]$.

- Possible relaxations of the above boundary conditions have been considered recently in the literature allowing for variations of the transverse boundary metric. These lead to enhancement of the asymptotic group with $\operatorname{Diff}\left(S^{2}\right)$ superrotations [26, 30-32] and/or Weyl rescaling symmetries [5, 22, 33, 34] (see also [35] for a review). While the case that we discuss here is the most natural to study the celestial holography since it readily implies the conformal symmetries on the celestial Riemann surface, we will comment on these extensions in the discussion section.

- In the above conditions, we set the order $r^{0}$ in the expansion of $g_{A B}$ to zero. Turning on this term would bring some $\log r$ terms in the expansion that we want to avoid [5,36,37]. For discussions on polyhomogeneous spacetimes, see e.g. [38-45].

Solving Einstein's equations in vaccuum with vanishing cosmological constant for the boundary conditions (2.3) yields the following expansions [5, 36]:

$$
\begin{aligned}
\frac{V}{r} & =-1+\frac{2 M}{r}+\mathcal{O}\left(r^{-2}\right), \quad \beta=\frac{1}{r^{2}}\left[-\frac{1}{32} C^{A B} C_{A B}\right]+\mathcal{O}\left(r^{-3}\right), \\
g_{A B} & =r^{2} \stackrel{\leftrightarrow}{q}_{A B}+r C_{A B}+\mathcal{O}\left(r^{-1}\right), \\
U^{A} & =-\frac{1}{2 r^{2}} D_{B} C^{A B}-\frac{2}{3} \frac{1}{r^{3}}\left[N^{A}-\frac{1}{2} C^{A B} D^{C} C_{B C}\right]+\mathcal{O}\left(r^{-4}\right),
\end{aligned}
$$

where $M=M(u, x)$ is the Bondi mass aspect, $N_{A}=N_{A}(u, x)$ is the angular momentum aspect. The 2 -sphere indices in (2.5) are lowered an raised with $\stackrel{\circ}{q}_{A B}$ and its inverse, and $D_{A}$ is the Levi-Civita connection on the celestial Riemann surface associated to $\stackrel{\circ}{A B}_{A B}$. The Bondi mass and angular momentum aspects satisfy the time evolution equations

$$
\begin{aligned}
\partial_{u} M= & -\frac{1}{8} N_{A B} N^{A B}+\frac{1}{4} D_{A} D_{B} N^{A B} \\
\partial_{u} N_{A}= & D_{A} M+\frac{1}{16} D_{A}\left(N_{B C} C^{B C}\right)-\frac{1}{4} N^{B C} D_{A} C_{B C}-\frac{1}{4} D_{B}\left(C^{B C} N_{A C}-N^{B C} C_{A C}\right) \\
& -\frac{1}{4} D_{B} D^{B} D^{C} C_{A C}+\frac{1}{4} D_{B} D_{A} D_{C} C^{B C},
\end{aligned}
$$

with $N_{A B}=\partial_{u} C_{A B}$ the Bondi news tensor. 
The residual diffeomorphisms that preserve the Bondi gauge (2.1) and falloff conditions (2.3) are generated by vectors fields $\xi=\xi^{u} \partial_{u}+\xi^{z} \partial+\xi^{\bar{z}} \bar{\partial}+\xi^{r} \partial_{r}$ whose components read as

$$
\begin{aligned}
& \xi^{u}=\left(\Omega_{S} \bar{\Omega}_{S}\right)^{-\frac{1}{2}} \mathcal{T}+\frac{u}{2}\left(D_{z} \mathcal{Y}+D_{\bar{z}} \overline{\mathcal{Y}}\right) \\
& \xi^{z}=\mathcal{Y}+\mathcal{O}\left(r^{-1}\right) \quad \xi^{\bar{z}}=\overline{\mathcal{Y}}+\mathcal{O}\left(r^{-1}\right) \\
& \xi^{r}=-\frac{r}{2}\left(D_{z} \mathcal{Y}+D_{\bar{z}} \overline{\mathcal{Y}}\right)+\mathcal{O}\left(r^{0}\right)
\end{aligned}
$$

where $\mathcal{T}=\mathcal{T}(z, \bar{z})$ is the supertranslation parameter and $\mathcal{Y}=\mathcal{Y}(z), \overline{\mathcal{Y}}=\overline{\mathcal{Y}}(\bar{z})$ are the superrotation parameters satisfying the conformal Killing equation

$$
D_{\bar{z}} \mathcal{Y}=0, \quad D_{z} \overline{\mathcal{Y}}=0 .
$$

Using the modified Lie bracket $\left[\xi_{1}, \xi_{2}\right]_{\star}=\left[\xi_{1}, \xi_{2}\right]-\delta_{\xi_{1}} \xi_{2}+\delta_{\xi_{2}} \xi_{1}$ where the last two terms take into account the field-dependence of the asymptotic Killing vectors (2.7) at subleading order in $r$ [5, 46], the asymptotic Killing vectors (2.7) satisfy the commutation relations

$$
\left[\xi\left(\mathcal{T}_{1}, \mathcal{Y}_{1}, \overline{\mathcal{Y}}_{1}\right), \xi\left(\mathcal{T}_{2}, \mathcal{Y}_{2}, \overline{\mathcal{Y}}_{2}\right)\right]_{\star}=\xi\left(\mathcal{T}_{12}, \mathcal{Y}_{12}, \overline{\mathcal{Y}}_{12}\right)
$$

with

$$
\mathcal{T}_{12}=\mathcal{Y}_{1} \partial \mathcal{T}_{2}-\frac{1}{2} \partial \mathcal{Y}_{1} \mathcal{T}_{2}-(1 \leftrightarrow 2)+c . c ., \quad \mathcal{Y}_{12}=\mathcal{Y}_{1} \partial \mathcal{Y}_{2}-(1 \leftrightarrow 2), \quad \overline{\mathcal{Y}}_{12}=\overline{\mathcal{Y}}_{1} \bar{\partial} \overline{\mathcal{Y}}_{2}-(1 \leftrightarrow 2)
$$

where c.c. stands for complex conjugate terms. This corresponds to the extended BMS algebra, namely

$$
\mathfrak{b m s}_{4}=(\mathrm{Witt} \oplus \overline{\mathrm{Witt}}) \oplus \mathfrak{s}^{*}
$$

where $\mathfrak{s}^{*}$ stands for the (possibly singular) supertranslations.

For convenience, we introduce the notations $f=\left(\Omega_{S} \bar{\Omega}_{S}\right)^{-\frac{1}{2}} \mathcal{T}+\frac{u}{2}\left(D_{z} \mathcal{Y}+D_{\bar{z}} \overline{\mathcal{Y}}\right)$ and $Y^{A}=(\mathcal{Y}, \overline{\mathcal{Y}})$. Under residual gauge diffeomorphisms $(2.7)$, the solution space transforms infinitesimally as

$$
\begin{aligned}
\delta_{(f, Y)} C_{A B}= & {\left[f \partial_{u}+\mathcal{L}_{Y}-\frac{1}{2} D_{C} Y^{C}\right] C_{A B}-2 D_{A} D_{B} f+\stackrel{\circ}{A}_{A B} D_{C} D^{C} f, } \\
\delta_{(f, Y)} N_{A B}= & {\left[f \partial_{u}+\mathcal{L}_{Y}\right] N_{A B}-\left(D_{A} D_{B} D_{C} Y^{C}-\frac{1}{2} \stackrel{\circ}{A B}_{A} D_{C} D^{C} D_{D} Y^{D}\right), } \\
\delta_{(f, Y)} M= & {\left[f \partial_{u}+\mathcal{L}_{Y}+\frac{3}{2} D_{C} Y^{C}\right] M } \\
& +\frac{1}{8} D_{C} D_{B} D_{A} Y^{A} C^{B C}+\frac{1}{4} N^{A B} D_{A} D_{B} f+\frac{1}{2} D_{A} f D_{B} N^{A B}, \\
\delta_{(f, Y)} N_{A}= & {\left[f \partial_{u}+\mathcal{L}_{Y}+D_{C} Y^{C}\right] N_{A}+3 M D_{A} f-\frac{3}{16} D_{A} f N_{B C} C^{B C} } \\
& -\frac{1}{32} D_{A} D_{B} Y^{B} C_{C D} C^{C D}+\frac{1}{4}\left(2 D^{B} f+D^{B} D_{C} D^{C} f\right) C_{A B} \\
& -\frac{3}{4} D_{B} f\left(D^{B} D^{C} C_{A C}-D_{A} D_{C} C^{B C}\right)+\frac{3}{8} D_{A}\left(D_{C} D_{B} f C^{B C}\right) \\
& +\frac{1}{2}\left(D_{A} D_{B} f-\frac{1}{2} D_{C} D^{C} f \stackrel{\circ}{A B}_{A B}\right) D_{C} C^{B C}+\frac{1}{2} D_{B} f N^{B C} C_{A C} .
\end{aligned}
$$


As one can see from the second expression above, the Bondi news $N_{A B}$ transforms inhomogeneously under superrotations. As discussed in [26], one can define the physical news $\hat{N}_{A B}$ as

$$
\hat{N}_{A B}(u, x)=N_{A B}(u, x)-N_{A B}^{v a c}(x),
$$

with

$$
N_{A B}^{v a c}(x)=\left[\frac{1}{2} D_{A} \Phi D_{B} \Phi-D_{A} D_{B} \Phi\right]^{T F}, \quad \Phi(z, \bar{z})=\varphi(z)+\bar{\varphi}(\bar{z})+\ln \left(\Omega_{S} \bar{\Omega}_{S}\right),
$$

where $T F$ stands for the trace-free part. $N_{A B}^{v a c}$ is the stress-tensor for a $2 d$ Euclidean Liouville theory living on the celestial Riemann surface with Lagrangian

$$
L[\Phi]=\sqrt{\stackrel{\circ}{q}}\left(\frac{1}{2} D_{A} \Phi D^{A} \Phi+\stackrel{\circ}{R} \Phi\right) .
$$

The Liouville scalar field $\Phi$ is called the "superboost field" and it encodes the refraction/velocity kick memory effects [26]. It satisfies the equation of motion

$$
\square \Phi=\stackrel{\circ}{R}=2 \Omega_{S} \bar{\Omega}_{S} \partial \bar{\partial} \ln \left(\Omega_{S} \bar{\Omega}_{S}\right)=2
$$

and transforms as

$$
\delta_{(f, Y)} \Phi=Y^{A} D_{A} \Phi+D_{A} Y^{A}
$$

or, equivalently, $\delta_{(f, Y)} \varphi=\mathcal{Y} \partial \varphi+\partial \mathcal{Y}, \delta_{(f, Y)} \bar{\varphi}=\overline{\mathcal{Y}} \bar{\partial} \bar{\varphi}+\bar{\partial} \overline{\mathcal{Y}}$, under residual gauge diffeomorphisms (2.7). Notice that the Liouville equation (2.16) is consistent with the action of the symmetries since $\delta_{f, Y}(\square \Phi-\stackrel{\circ}{R})=\left(\mathcal{L}_{Y}+D_{A} Y^{A}\right)(\square \Phi-\stackrel{\circ}{R})$. As a consequence of $(2.16)$ and (2.17), the Liouville stress-tensor (2.14) satisfies $D^{A} N_{A B}^{v a c}=0$ and transforms as

$$
\delta_{(f, Y)} N_{A B}^{v a c}=\mathcal{L}_{Y} N_{A B}^{v a c}-\left(D_{A} D_{B} D_{C} Y^{C}\right)^{T F} .
$$

One can show that it is related to the trace-free part of the Geroch tensor $\rho_{A B}[24,47-49]$. The interest of the physical news (2.13) is that it transforms homogeneously, i.e.

$$
\delta_{(f, Y)} \hat{N}_{A B}=\left[f \partial_{u}+\mathcal{L}_{Y}\right] \hat{N}_{A B},
$$

so that $\hat{N}_{A B}=0$ is a meaningful condition to impose in presence of superrotations to define non-radiative spacetimes.

In addition to the boundary conditions (2.3), one also imposes the following falloff conditions when $u \rightarrow \pm \infty$ that are compatible with the action of superrotations [24-26, 50]:

$$
N_{A B}=N_{A B}^{v a c}+o\left(u^{-2}\right), \quad C_{A B}=\left(u+C_{ \pm}\right) N_{A B}^{v a c}-2\left(D_{A} D_{B} C_{ \pm}\right)^{T F}+o\left(u^{-1}\right),
$$

where $C_{ \pm}$correspond to the values of the supertranslation field at $\mathscr{I}_{ \pm}^{+}$that encodes the displacement memory effect [51]. We have the transformation

$$
\delta_{(f, Y)} C_{ \pm}=\left(\Omega_{S} \bar{\Omega}_{S}\right)^{-\frac{1}{2}} \mathcal{T}+Y^{A} \partial_{A} C_{ \pm}-\frac{1}{2} D_{A} Y^{A} C_{ \pm} .
$$

As discussed in [24, 50], the falloffs (2.20) are stronger than those considered in e.g. [26], but we found that they are necessary for the finiteness of the flux related to superrotations 
that we will introduce in section 4 . The falloffs (2.20) imply that, at the corners $\mathscr{I}_{ \pm}^{+}$, the spacetime is non-radiative $\left(\left.\hat{N}_{A B}\right|_{\mathscr{V}_{ \pm}^{+}}=0\right)$ and the physical asymptotic shear defined by $\hat{C}_{A B}=C_{A B}-u N_{A B}^{v a c}$ is purely electric, i.e.

$$
\left.\left[\left(D_{B} D_{C}-\frac{1}{2} N_{B C}^{v a c}\right) \hat{C}_{A}^{C}-\left(D_{A} D_{C}-\frac{1}{2} N_{A C}^{v a c}\right) \hat{C}_{B}^{C}\right]\right|_{\mathscr{I}_{ \pm}^{+}}=0 .
$$

One can check that this condition is preserved under BMS transformations. It generalizes the standard electricity condition considered e.g. in [6] in presence of superrotations [24-26].

\section{Conformal fields on the celestial Riemann surface}

We now set up the stage for the boundary side of the celestial holography framework. From the previous section, we infer that the celestial Riemannian surface $\mathcal{S} \simeq \mathscr{I}^{+} / \mathbb{R}$ can be taken as the 2-punctured Riemann sphere endowed with the fixed Euclidian metric (2.4) [18]. It is convenient to complexify $\mathcal{S}$ and treat the coordinates $z$ and $\bar{z}$ independently. From the boundary point of view, one can consider the "extended conformal transformations" that preserve the conformal class of the metric (2.4). They are defined as the combined action of conformal coordinate transformations $z^{\prime}=z^{\prime}(z)$ and $\bar{z}^{\prime}=\bar{z}^{\prime}(\bar{z})$ and Weyl rescalings that induce the following transformations on the conformal factor:

$$
(\Omega \bar{\Omega})^{\prime}\left(z^{\prime}, \bar{z}^{\prime}\right)=(\Omega \bar{\Omega})(z, \bar{z})\left(\frac{\partial z^{\prime}}{\partial z}\right)\left(\frac{\partial \bar{z}^{\prime}}{\partial \bar{z}}\right) e^{-2 E_{R}\left(z^{\prime}, \bar{z}^{\prime}\right)},
$$

where $E_{R}(z, \bar{z})$ is the real Weyl rescaling parameter. ${ }^{1}$ These transformations preserve the particular representative (2.4) of the conformal class provided

$$
e^{E_{R}\left(z^{\prime}, \bar{z}^{\prime}\right)}=\frac{(1+z \bar{z})}{\left(1+z^{\prime} \bar{z}^{\prime}\right)} \sqrt{\left(\frac{\partial z^{\prime}}{\partial z}\right)\left(\frac{\partial \bar{z}^{\prime}}{\partial \bar{z}}\right)} .
$$

Infinitesimally, the extended conformal transformations (3.1) satisfying (3.2) are generated by the conformal Killing vectors $\mathcal{Y}(z) \partial+\overline{\mathcal{Y}}(\bar{z}) \bar{\partial}$ induced on $\mathcal{S}$ from the bulk superrotations defined in (2.7).

A conformal field of weights $(h, \bar{h})$ is defined as a field $\phi_{h, \bar{h}}(x)$ on $\mathcal{S}$ which transforms as

$$
\phi_{h, \bar{h}}^{\prime}\left(x^{\prime}\right)=\left(\frac{\partial z}{\partial z^{\prime}}\right)^{h}\left(\frac{\partial \bar{z}}{\partial \bar{z}^{\prime}}\right)^{\bar{h}} \phi_{h, \bar{h}}(x)
$$

under transformations (3.1) with the constraint (3.2). One can then define a spin weight $J$ and a boost weight/conformal dimension $\Delta$ as usual:

$$
J=h-\bar{h}, \quad \Delta=(h+\bar{h}) .
$$

As explained in $[18,33]$, there is a one-to-one map between conformal fields and weighted scalars with spin weight $s=J$ and boost weight $w=-\Delta$. The weighted scalar point of

\footnotetext{
${ }^{1}$ The parametrization of the Weyl rescaling in (3.1) is precisely the one considered in [18, 22, 33]. It is related to the parametrization used in $[5,34]$ through a redefinition of the parameters.
} 
view is the one that naturally arises when starting from the solution space of gravity [5254]. However, it can be easily related to the conformal field point of view by using the conformal factor of the metric. In the present paper, we choose to work in the latter framework which is more adapted to celestial holography.

It will turn out to be useful to introduce the following derivative operators:

$$
\mathscr{D} \phi_{h, \bar{h}}=\left[D_{z}-h \partial \Phi\right] \phi_{h, \bar{h}}, \quad \overline{\mathscr{D}} \phi_{h, \bar{h}}=\left[D_{\bar{z}}-\bar{h} \bar{\partial} \Phi\right] \phi_{h, \bar{h}},
$$

which act on $(h, \bar{h})$ conformal fields to give conformal fields of weights $(h+1, \bar{h})$ and $(h, \bar{h}+1)$, respectively. They satisfy $\mathscr{D}\left(\Omega_{S} \bar{\Omega}_{S}\right)=0=\overline{\mathscr{D}}\left(\Omega_{S} \bar{\Omega}_{S}\right)$. These operators coincide with the Weyl covariant derivative operators introduced in [18] for the framework that we are considering here; i.e. with fixed representative (2.4). In particular, the Liouville field introduced in (2.14) naturally arises as part of the Weyl connection. Furthermore, the operators (3.5) also correspond to the Witt $\oplus \overline{\mathrm{Witt}}$ version of the $\operatorname{Diff}\left(S^{2}\right)$-covariant derivative introduced in [24] (see also [50]). One can check that

$$
[\mathscr{D}, \overline{\mathscr{D}}] \phi_{h, \bar{h}}=0 \text {. }
$$

We assume that the conformal fields can be expanded in formal series as

$$
\phi_{h, \bar{h}}(z, \bar{z})=\sum_{k, l} a_{k, l} h, \bar{h} Z_{k, l}, \quad{ }_{h, \bar{h}} Z_{k, l}=z^{-h-k} \bar{z}^{-\bar{h}-l}
$$

where the coefficients $a_{k, l} \in \mathbb{C}$ satisfy appropriate conditions $[55,56]$. If $h, \bar{h}$ are integers (resp. half integers), then $k, l$ are taken to be integers (resp. half integers). The residues of $\phi_{h, \bar{h}}(z, \bar{z})$ with respect to $z$ and $\bar{z}$ are defined as

$$
\operatorname{Res}_{z}\left[\phi_{h, \bar{h}}(z, \bar{z})\right]=\sum_{l} a_{1-h, l} \bar{z}^{-\bar{h}-l}, \quad \operatorname{Res}_{\bar{z}}\left[\phi_{h, \bar{h}}(z, \bar{z})\right]=\sum_{k} a_{k, 1-\bar{h}} z^{-h-k} .
$$

From the residue theorem, we have the fundamental relations

$$
\oint_{\mathcal{C}} \frac{d z}{2 i \pi} \phi_{h, \bar{h}}(z, \bar{z})=\operatorname{Res}_{z}\left[\phi_{h, \bar{h}}(z, \bar{z})\right], \quad \oint_{\mathcal{C}} \frac{d \bar{z}}{2 i \pi} \phi_{h, \bar{h}}(z, \bar{z})=\operatorname{Res}_{\bar{z}}\left[\phi_{h, \bar{h}}(z, \bar{z})\right]
$$

where $\mathcal{C}$ is a contour around the puncture. Notice that total derivative terms can be discarded in the contour integrals since there is no $\log z$ terms in the expansion (3.7). We use the notation $\int_{\mathcal{S}} \frac{d z d \bar{z}}{(2 i \pi)^{2}}=\oint_{\mathcal{C}} \frac{d z}{2 i \pi} \int_{\mathcal{C}} \frac{d \bar{z}}{2 i \pi}$ to designate the integral over the celestial Riemann surface $\mathcal{S} .^{2}$ The expansion (3.7) is inverted by the relation

$$
a_{k, l}=\int_{\mathcal{S}} \frac{d z d \bar{z}}{(2 i \pi)^{2}} z^{k+h-1} \bar{z}^{l+\bar{h}-1} \phi_{h, \bar{h}}(z, \bar{z})
$$

\footnotetext{
${ }^{2}$ Notice that the standard normalization for the measure on the celestial sphere is such that

$$
\int_{\mathcal{S}} i d z d \bar{z}\left(\Omega_{S} \bar{\Omega}_{S}\right)^{-1}=\int_{0}^{\pi} d \theta \sin ^{2} \theta \int_{0}^{2 \pi} d \phi=4 \pi
$$

when using stereographic coordinates $z=\cot \left(\frac{\theta}{2}\right) e^{-i \phi}, \bar{z}=\cot \left(\frac{\theta}{2}\right) e^{i \phi}$.
} 
The formal Dirac delta-functions are defined as the following formal distributions $[55,56]$ :

$$
\delta(z-w)=z^{-1} \sum_{k \in \mathbb{Z}}\left(\frac{z}{w}\right)^{k}, \quad \delta(\bar{z}-\bar{w})=\bar{z}^{-1} \sum_{k \in \mathbb{Z}}\left(\frac{\bar{z}}{\bar{w}}\right)^{k} .
$$

They satisfy the useful properties $\oint_{\mathcal{C}} \frac{d z}{2 i \pi} \delta(z-w) \phi_{h, \bar{h}}(z, \bar{z})=\phi_{h, \bar{h}}(w, \bar{z}), \delta(z-w)=\delta(w-z)$, $(z-w) \delta(z-w)=0, \partial_{z} \delta(z-w)=-\partial_{w} \delta(z-w)$, together with the corresponding relations for $\delta(\bar{z}-\bar{w})$. We write the delta-function on the celestial Riemann surface as

$$
\delta^{2}(z-w)=\delta(z-w) \delta(\bar{z}-\bar{w}),
$$

so that $\int_{\mathcal{S}} \frac{d z d \bar{z}}{(2 i \pi)^{2}} \phi_{h, \bar{h}}(z, \bar{z}) \delta^{2}(z-w)=\phi_{h, \bar{h}}(w, \bar{w})$.

\section{Generators and momenta}

We now identify the parameters of the extended BMS algebra and some non-local combinations of the solution space introduced in section 2 from bulk considerations, as conformal fields on the celestial Riemann surface in the sense of section 3. The conformal weights of the various fields discussed in this paper are summarized in table 1.

Let us start with the $\mathfrak{b m s}_{4}$ symmetry parameter given in equation $(2.7){ }^{3}$ The supertranslation parameters $\mathcal{T}(z, \bar{z})$ can be seen as real conformal fields of weights $\left(-\frac{1}{2},-\frac{1}{2}\right)[18] .{ }^{4}$ They can be expanded in formal series as in (3.7):

$$
\mathcal{T}(z, \bar{z})=\sum_{k, l} t_{k, l} \mathscr{T}_{k, l}, \quad \mathscr{T}_{k, l}={ }_{-\frac{1}{2},-\frac{1}{2}} Z_{k, l}=z^{\frac{1}{2}-k} \bar{z}^{\frac{1}{2}-l},
$$

with $k, l$ half-integers and $\bar{t}_{k, l}=t_{l, k}$ so that $\mathcal{T}$ is real. Notice that the four Poincaré translations are spanned by $\mathscr{T}_{\frac{1}{2}, \frac{1}{2}}, \mathscr{T}_{\frac{1}{2},-\frac{1}{2}}, \mathscr{T}_{-\frac{1}{2}, \frac{1}{2}}$ and $\mathscr{T}_{-\frac{1}{2},-\frac{1}{2}}$.

Superrotations are parametrized by the complex conformal fields $\mathcal{Y}(z), \overline{\mathcal{Y}}(\bar{z})$ of weights $(-1,0)$ and $(0,-1)$, respectively [18]. The infinitesimal actions of superrotations on a conformal field $\phi_{h, \bar{h}}(z, \bar{z})$ are given by

$$
\delta \mathcal{Y} \phi_{h, \bar{h}}=\mathcal{Y} \partial \phi_{h, \bar{h}}+h \partial \mathcal{Y} \phi_{h, \bar{h}}, \quad \delta_{\overline{\mathcal{Y}}} \phi_{h, \bar{h}}=\overline{\mathcal{Y}} \bar{\partial} \phi_{h, \bar{h}}+\bar{h} \bar{\partial} \overline{\mathcal{Y}} \phi_{h, \bar{h}}
$$

These are the infinitesimal analogues of (3.3). Superrotation vector fields can be expanded as in (3.7):

$$
\mathcal{Y}(z)=\sum_{m} y_{m} \mathcal{Y}_{m}, \quad \mathcal{Y}_{m}=z^{1-m}, \quad \overline{\mathcal{Y}}(\bar{z})=\sum_{m} \bar{y}_{m} \overline{\mathcal{Y}}_{m}, \quad \overline{\mathcal{Y}}_{m}=\bar{z}^{1-m}
$$

where $m \in \mathbb{Z}$ and $y_{m}, \bar{y}_{m}$ are complex numbers. The six global Lorentz parameters are spanned by $\mathcal{Y}_{-1}, \mathcal{Y}_{0}, \mathcal{Y}_{1}$, and their complex conjugates. In terms of (4.3), equation (4.2) can be rewritten as

$$
\delta \mathcal{Y}_{m} \phi_{h, \bar{h}}=z^{1-m} \partial \phi_{h, \bar{h}}+h(1-m) z^{-m} \phi_{h, \bar{h}}, \quad \delta_{\overline{\mathcal{Y}}_{m}} \phi_{h, \bar{h}}=\bar{z}^{1-m} \bar{\partial} \phi_{h, \bar{h}}+\bar{h}(1-m) \bar{z}^{-m} \phi_{h, \bar{h}}
$$

\footnotetext{
${ }^{3}$ In this paper, since we are focusing on the conformal field point of view, we do not write the " " notation above the conformal fields, which contrasts with the convention used in [18].

${ }^{4}$ The choice of conformal weights for the symmetry parameters will be justified later through the pairing between generators and momenta in (5.1).
} 
or, equivalently,

$$
\delta_{\mathcal{Y}_{m}}\left({ }_{h, \bar{h}} Z_{k, l}\right)=-(k+h m)_{h, \bar{h}} Z_{m+k, l}, \quad \delta_{\overline{\mathcal{Y}}_{m}}\left({ }_{h, \bar{h}} Z_{k, l}\right)=-(l+\bar{h} m)_{h, \bar{h}} Z_{k, l+m} .
$$

For convenience, the $\mathfrak{b m s}_{4}$ commutation relations (2.9) and (2.10) can be rewritten using the notations $\xi(\mathcal{T}, 0,0) \rightarrow \mathcal{T}, \xi(0, \mathcal{Y}, 0) \rightarrow \mathcal{Y}, \xi(0,0, \overline{\mathcal{Y}}) \rightarrow \overline{\mathcal{Y}},[., .]_{\star} \rightarrow[.,$.$] . We have$

$$
\begin{array}{lll}
{\left[\mathcal{Y}_{1}, \mathcal{Y}_{2}\right]=\mathcal{Y}_{1} \partial \mathcal{Y}_{2}-\mathcal{Y}_{2} \partial \mathcal{Y}_{1},} & {\left[\overline{\mathcal{Y}}_{1}, \overline{\mathcal{Y}}_{2}\right]=\overline{\mathcal{Y}}_{1} \bar{\partial} \overline{\mathcal{Y}}_{2}-\overline{\mathcal{Y}}_{2} \bar{\partial} \overline{\mathcal{Y}}_{1},} & {\left[\mathcal{T}_{1}, \mathcal{T}_{2}\right]=0,} \\
{\left[\mathcal{Y}_{1}, \mathcal{T}_{2}\right]=\mathcal{Y}_{1} \partial \mathcal{T}_{2}-\frac{1}{2} \partial \mathcal{Y}_{1} \mathcal{T}_{2},} & {\left[\overline{\mathcal{Y}}_{1}, \mathcal{T}_{2}\right]=\overline{\mathcal{Y}}_{1} \bar{\partial} \mathcal{T}_{2}-\frac{1}{2} \bar{\partial} \overline{\mathcal{Y}}_{1} \mathcal{T}_{2} .}
\end{array}
$$

In terms of expansions (4.1) and (4.3), these commutation relations become $[5,57]$

$$
\begin{aligned}
{\left[\mathcal{Y}_{m}, \mathcal{Y}_{n}\right] } & =(m-n) \mathcal{Y}_{m+n}, & {\left[\overline{\mathcal{Y}}_{m}, \overline{\mathcal{Y}}_{n}\right] } & =(m-n) \overline{\mathcal{Y}}_{m+n}, \\
{\left[\mathcal{Y}_{m}, \mathscr{T}_{k, l}\right] } & =\left(\frac{1}{2} m-k\right) \mathscr{T}_{m+k, l}, & {\left[\overline{\mathcal{Y}}_{m}, \mathscr{T}_{k, l}\right] } & =\left(\frac{1}{2} m-l\right) \mathscr{T}_{k, m+l}, \\
{\left[\mathcal{Y}_{m}, \overline{\mathcal{Y}}_{n}\right] } & =0=\left[\mathscr{T}_{k, l}, \mathscr{T}_{r, s}\right] . & &
\end{aligned}
$$

Let us now define the BMS momentum fluxes as particular non-local combinations of the solution space data of asymptotically flat spacetimes appearing in (2.5) and interpret them as conformal fields on the celestial Riemann surface $\mathcal{S}$.

First, the supermomentum flux $\mathcal{P}(z, \bar{z})$ is defined by

$$
\mathcal{P}=\frac{1}{4 \pi G} \int_{-\infty}^{+\infty} d u \partial_{u} \mathcal{M}=\left.\frac{\mathcal{M}}{4 \pi G}\right|_{\mathscr{I}_{-}^{+}} ^{\mathscr{I}_{+}^{+}}, \quad \mathcal{M}=\left(\Omega_{S} \bar{\Omega}_{S}\right)^{-\frac{3}{2}}\left[M+\frac{1}{8}\left(C_{z z} N_{v a c}^{z z}+C_{\bar{z} \bar{z}} N_{v a c}^{\bar{z} \bar{z}}\right)\right]
$$

which corresponds to the difference of values of the supermomentum $\mathcal{M}(u, z, \bar{z})$ between the two non-radiative asymptotic regions $\mathscr{I}_{ \pm}^{+}$. Here we used the prescription of $[25,26]$ to define the supermomentum in terms of the solution space. The supermomentum flux $\mathcal{P}(z, \bar{z})$ can be seen as a $(J=0)$ conformal field of weights $\left(\frac{3}{2}, \frac{3}{2}\right)$. Indeed, under infinitesimal BMS transformations, one can deduce from (2.12) and (2.20) that

$$
\delta_{(\mathcal{T}, \mathcal{Y}, \overline{\mathcal{Y}})} \mathcal{P}=\left[\mathcal{Y} \partial+\overline{\mathcal{Y}} \bar{\partial}+\frac{3}{2} \partial \mathcal{Y}+\frac{3}{2} \bar{\partial} \overline{\mathcal{Y}}\right] \mathcal{P}
$$

which is the expected infinitesimal transformation law for a $\left(\frac{3}{2}, \frac{3}{2}\right)$ conformal field (see (4.2)). For later purposes, it is useful to split the supermomentum flux into soft and hard parts involving linear, respectively quadratic, terms in $\hat{N}_{A B}$ inside the integral. We prescribe

$$
\mathcal{P}=\mathcal{P}_{\text {soft }}+\mathcal{P}_{\text {hard }}
$$

with

$$
\begin{aligned}
\mathcal{P}_{\text {soft }} & =\frac{1}{16 \pi G} \int_{-\infty}^{+\infty} d u\left(\Omega_{S} \bar{\Omega}_{S}\right)^{-\frac{3}{2}}\left[\left(D_{z}^{2}-\frac{1}{2} N_{z z}^{v a c}\right) \hat{N}^{z z}+\left(D_{\bar{z}}^{2}-\frac{1}{2} N_{\bar{z} \bar{z}}^{v a c}\right) \hat{N}^{\bar{z} \bar{z}}\right], \\
\mathcal{P}_{\text {hard }} & =-\frac{1}{16 \pi G} \int_{-\infty}^{+\infty} d u\left(\Omega_{S} \bar{\Omega}_{S}\right)^{-\frac{3}{2}}\left[\hat{N}_{z z} \hat{N}^{z z}\right] .
\end{aligned}
$$


Notice that the fluxes of supermomenta defined as in (4.11) are finite in $u$ and vanish in stationary configurations where $\hat{N}_{A B}=0$, which is a desirable physical requirement [19, 26]. One can show that the soft and the hard parts transform separately as in (4.9), namely

$$
\delta_{(\mathcal{T}, \mathcal{Y}, \overline{\mathcal{Y}})} \mathcal{P}_{\text {soft } / \text { hard }}=\left[\mathcal{Y} \partial+\overline{\mathcal{Y}} \bar{\partial}+\frac{3}{2} \partial \mathcal{Y}+\frac{3}{2} \bar{\partial} \overline{\mathcal{Y}}\right] \mathcal{P}_{\text {soft } / \text { hard }}
$$

They can therefore be both interpreted as conformal fields of weights $\left(\frac{3}{2}, \frac{3}{2}\right)$, which justifies the specific split between hard and soft parts in (4.11). In terms of the superrotationcovariant derivative operators introduced in (3.5), the soft part can be elegantly rewritten as

$$
\mathcal{P}_{\text {soft }}=\mathscr{D}^{2} \overline{\mathscr{N}}^{(0)}+\overline{\mathscr{D}}^{2} \mathscr{N}^{(0)}, \quad \mathscr{N}^{(0)}=\frac{1}{16 \pi G} \int_{-\infty}^{+\infty} d u\left(\Omega_{S} \bar{\Omega}_{S}\right)^{\frac{1}{2}} \hat{N}_{z z},
$$

where the leading soft mode of the news tensor $\mathscr{N}^{(0)}(z, \bar{z})$ is a $\left(\frac{3}{2},-\frac{1}{2}\right)$ conformal field. Moreover, using the electricity condition (2.22) encoded in the falloffs (2.20), we have $\mathscr{D}^{2} \overline{\mathscr{N}}^{(0)}=\overline{\mathscr{D}}^{2} \mathscr{N}^{(0)}$ and

$$
\begin{aligned}
\mathcal{P}_{\text {soft }} & =2 \mathscr{D}^{2} \overline{\mathscr{N}}^{(0)} \\
& =\frac{1}{8 \pi G}\left(\Omega_{S} \bar{\Omega}_{S}\right)^{\frac{1}{2}}\left(D_{z}^{2}-\frac{1}{2} N_{z z}^{v a c}\right)\left[\Delta C N_{\bar{z} \bar{z}}^{v a c}-2 D_{\bar{z}}^{2} \Delta C\right],
\end{aligned}
$$

with $\Delta C=C_{+}-C_{-}$the difference of the supertranslation field between the future and past corners of $\mathscr{I}^{+}$.

Second, the super angular momentum flux is parametrized by the equivalence classes $[\mathcal{J}]$ and $[\overline{\mathcal{J}}]$ for the equivalence relation $[18]$

$$
\mathcal{J} \equiv \mathcal{J}+\mathscr{D} \mathcal{L}, \quad \overline{\mathcal{J}} \equiv \overline{\mathcal{J}}+\overline{\mathscr{D}} \overline{\mathcal{L}}
$$

These equivalence classes can be seen as complex conformal fields of weights $(1,2)$ and $(2,1)$, respectively. In terms of the gravitational data, we define

$$
\mathcal{J}=\frac{1}{8 \pi G} \int_{-\infty}^{+\infty} d u \partial_{u} \mathcal{N}=\left.\frac{\mathcal{N}}{8 \pi G}\right|_{\mathscr{I}_{-}^{+}} ^{\mathscr{I}_{+}^{+}}
$$

which corresponds to the difference of values between $\mathscr{I}_{ \pm}^{+}$of the super angular momentum $\mathcal{N}(u, z, \bar{z})$ defined by

$$
\begin{aligned}
\mathcal{N}= & \left(\Omega_{S} \bar{\Omega}_{S}\right)^{-1}\left[N_{\bar{z}}-u \Omega_{S}^{3} D_{\bar{z}} \mathcal{M}+\frac{1}{4} C_{\bar{z} \bar{z}} D_{\bar{z}} C^{\bar{z} \bar{z}}+\frac{3}{16} D_{\bar{z}}\left(C_{z z} C^{z z}\right)\right] \\
& +\frac{u}{4}\left(\Omega_{S} \bar{\Omega}_{S}\right)^{-1} D^{z}\left[\left(D_{z}^{2}-\frac{1}{2} N_{z z}^{v a c}\right) C_{\bar{z}}^{z}-\left(D_{\bar{z}}^{2}-\frac{1}{2} N_{\bar{z} \bar{z}}^{v a c}\right) C_{z}^{\bar{z}}\right] .
\end{aligned}
$$

We have the analogous complex conjugate relations for $\overline{\mathcal{J}}(z, \bar{z})$ and $\overline{\mathcal{N}}(u, z, \bar{z})$. Here we used a prescription based on $[25,26,58]$ to define the super angular momentum. ${ }^{5}$ Under

\footnotetext{
${ }^{5}$ The prescription (4.17) for the super angular momentum differs from the one proposed in [25] by magnetic contributions of the shear that do not play any role at $\mathscr{I}_{ \pm}^{+}$, but that allow to have vanishing fluxes for stationary solutions $\hat{N}_{A B}=0$ (see [59] for a detailed discussion).
} 
infinitesimal BMS transformations acting through (2.12), the super angular momentum flux transforms as

$$
\delta_{(\mathcal{T}, \mathcal{Y}, \overline{\mathcal{Y}})} \mathcal{J}=\mathcal{Y} \partial \mathcal{J}+\overline{\mathcal{Y}} \overline{\mathcal{J}}+\partial \mathcal{Y} \mathcal{J}+2 \bar{\partial} \overline{\mathcal{Y}} \mathcal{J}+\frac{1}{2} \mathcal{T} \bar{\partial} \mathcal{P}+\frac{3}{2} \bar{\partial} \mathcal{T} \mathcal{P}
$$

together with the complex conjugate relation for $\overline{\mathcal{J}}$. For future purposes, it is useful to split the super angular momentum flux into soft and hard parts; we propose

$$
\mathcal{J}=\mathcal{J}_{\text {soft }}+\mathcal{J}_{\text {hard }}
$$

with

$$
\begin{aligned}
\mathcal{J}_{\text {soft }}=-\frac{1}{16 \pi G} \int_{-\infty}^{+\infty} & d u\left(\Omega_{S} \bar{\Omega}_{S}\right)^{-1}\left[u\left(D_{\bar{z}}^{3}-2 N_{\bar{z} \bar{z}}^{v a c} D_{\bar{z}}-D_{\bar{z}} N_{\bar{z} \bar{z}}^{v a c}\right) \hat{N}^{\bar{z} \bar{z}}\right. \\
& \left.+N^{\bar{z} \bar{z}} D_{\bar{z}}\left[\left(D_{\bar{z}}^{2}-\frac{1}{2} N_{\bar{z} \bar{z}}^{v a c}\right) C_{-}\right]+3 D_{\bar{z}} N^{\bar{z} \bar{z}}\left(D_{\bar{z}}^{2}-\frac{1}{2} N_{\bar{z} \bar{z}}^{v a c}\right) C_{-}\right] \\
\mathcal{J}_{\text {hard }}=\frac{1}{8 \pi G} \int_{-\infty}^{+\infty} d u & \left(\Omega_{S} \bar{\Omega}_{S}\right)^{-1}\left[\frac{3}{4} \hat{C}_{\bar{z} \bar{z}} D_{\bar{z}} \hat{N}^{\bar{z} \bar{z}}+\frac{1}{4} \hat{N}^{\bar{z} \bar{z}} D_{\bar{z}} \hat{C}_{\bar{z} \bar{z}}+\frac{u}{4} D_{\bar{z}}\left(\hat{N}_{\bar{z} \bar{z}} \hat{N}^{\bar{z} \bar{z}}\right)\right. \\
& \left.+\frac{1}{2} N^{\bar{z} \bar{z}} D_{\bar{z}}\left[\left(D_{\bar{z}}^{2}-\frac{1}{2} N_{\bar{z} \bar{z}}^{v a c}\right) C_{-}\right]+\frac{3}{2} D_{\bar{z}} N^{\bar{z} \bar{z}}\left(D_{\bar{z}}^{2}-\frac{1}{2} N_{\bar{z} \bar{z}}^{v a c}\right) C_{-}\right]
\end{aligned}
$$

and the complex conjugate relations for $\overline{\mathcal{J}}_{\text {soft }}$ and $\overline{\mathcal{J}}_{\text {hard }}$. Notice that the fluxes of super angular momenta (4.20) are finite thanks to the stronger $u$-falloffs that were taken (2.20) and vanish in stationary configurations where $\hat{N}_{A B}=0[19,26]$. One can show that the soft and the hard parts transform separately as in (4.18), namely

$$
\begin{aligned}
& \delta_{(\mathcal{T}, \mathcal{Y}, \overline{\mathcal{Y}})} \mathcal{J}_{\text {soft }}=\mathcal{Y} \partial \mathcal{J}_{\text {soft }}+\overline{\mathcal{Y}} \bar{\partial} \mathcal{J}_{\text {soft }}+2 \bar{\partial} \overline{\mathcal{Y}} \mathcal{J}_{\text {soft }}+\partial \mathcal{Y} \mathcal{J}_{\text {soft }}+\frac{1}{2} \mathcal{T} \bar{\partial} \mathcal{P}_{\text {soft }}+\frac{3}{2} \bar{\partial} \mathcal{T} \mathcal{P}_{\text {soft }}, \\
& \delta_{(\mathcal{T}, \mathcal{Y}, \overline{\mathcal{Y}})} \mathcal{J}_{\text {hard }}=\mathcal{Y} \partial \mathcal{J}_{\text {hard }}+\overline{\mathcal{Y}} \bar{\partial} \mathcal{J}_{\text {hard }}+2 \bar{\partial} \overline{\mathcal{Y}} \mathcal{J}_{\text {hard }}+\partial \mathcal{Y} \mathcal{J}_{\text {hard }}+\frac{1}{2} \mathcal{T} \bar{\partial} \mathcal{P}_{\text {hard }}+\frac{3}{2} \bar{\partial} \mathcal{T} \mathcal{P}_{\text {hard }},
\end{aligned}
$$

which implies that $\mathcal{J}_{\text {soft }}$ and $\mathcal{J}_{\text {hard }}\left(\overline{\mathcal{J}}_{\text {soft }}\right.$ and $\left.\overline{\mathcal{J}}_{\text {hard }}\right)$ can be seen separately as conformal fields of weights $(1,2)$ (respectively $(2,1)$ ). The transformations $(4.21)$ justify the specific choice of split prescribed between soft and hard parts in (4.20). In particular, the terms involving the supertranslation field $C_{-}$at $\mathscr{I}_{-}^{+}$ensure that the expressions transform as they should under supertranslations. ${ }^{6}$ In terms of the derivative operators (3.5), the soft part can be rewritten as

$$
\begin{aligned}
& \mathcal{J}_{\text {soft }}=-\overline{\mathscr{D}}^{3} \mathscr{N}^{(1)}-\overline{\mathscr{D}}^{3} \mathscr{C} \mathscr{N}^{(0)}-3 \overline{\mathscr{D}}^{2} \mathscr{C} \overline{\mathscr{D}}^{(0)}, \\
& \mathscr{N}^{(1)}=\frac{1}{16 \pi G} \int_{-\infty}^{+\infty} d u\left(\Omega_{S} \bar{\Omega}_{S}\right) u \hat{N}_{z z}, \quad \mathscr{C}=\left(\Omega_{S} \bar{\Omega}_{S}\right)^{\frac{1}{2}} C_{-},
\end{aligned}
$$

where the subleading soft mode of the news tensor $\mathscr{N}^{(1)}(z, \bar{z})$ is a $(1,-1)$ conformal field and $\mathscr{C}(z, \bar{z})$ is a $\left(-\frac{1}{2},-\frac{1}{2}\right)$ conformal field.

\footnotetext{
${ }^{6}$ One could have replaced $C_{-}$by $C_{+}$in the expressions (4.20) without affecting the result (4.21).
} 
As already mentioned, the prescription for the BMS momenta (4.8) and (4.17) that we are using here have all the desired properties, including finiteness in $u$, vanishing for nonradiative solutions, vanishing for vacuum configurations and closure under the standard bracket when considering the integrated fluxes over $\mathscr{I}^{+}$(see section 5). Concerning the splits between soft and hard parts in (4.11) and (4.20), they differ from those originally proposed in $[6,9,12]$ (see also [11]) by terms involving the memory fields $\varphi(z), \bar{\varphi}(\bar{z})$ and $C_{-}$that label the vacuum degeneracy [27-29]. When setting $\varphi=0=\bar{\varphi}\left(N_{A B}^{v a c}=0\right)$ and $C_{-}=0$, we consistently recover the standard expressions. The additional terms that we have allow us to obtain better transformation laws (4.12) and (4.21) under the action of BMS symmetries. As we will see in section 6 , this will be of major importance to identify the CCFT operators in the solution space of gravity that obey the desired constraints and transformation properties.

\section{BMS flux algebra}

When using covariant phase space methods [60-63], BMS surface charges are non-integrable due to the presence of radiation [19, 20]. Defining meaningful finite charges requires to impose additional criteria to isolate a specific integrable part (see e.g. [22, 23, 25, 26, 64$66]$ for recent proposals of such criteria and [67,68] for the implication of the various expressions on observational data). Here, we follow the prescription of $[25,58,59]$ (see also footnote 5) to select the integrable part and define the "finite" charges. The fluxes are then obtained by expressing the finite surface charge integrals as volume integrals over $\mathscr{I}^{+}$. In terms of the generators and flux of momenta that were introduced in section 4 , the BMS fluxes read as ${ }^{7}$

$$
F_{(\mathcal{T}, \mathcal{Y}, \overline{\mathcal{Y}})}=\int_{\mathcal{S}} \frac{d z d \bar{z}}{(2 i \pi)^{2}}[\mathcal{T} \mathcal{P}+\mathcal{Y} \overline{\mathcal{J}}+\overline{\mathcal{Y}} \mathcal{J}]=\frac{1}{8 \pi G} \int_{\mathscr{I}^{+}} d u \frac{d z d \bar{z}}{(2 i \pi)^{2}} \partial_{u}[2 \mathcal{T} \mathcal{M}+\mathcal{Y} \overline{\mathcal{N}}+\overline{\mathcal{Y}} \mathcal{N}]
$$

As mentioned above, the conformal weights of the BMS parameters $(\mathcal{T}, \mathcal{Y}, \overline{\mathcal{Y}})$ are completely determined by those of the measure $d z d \bar{z}$ and the momentum fluxes $(\mathcal{P},[\mathcal{J}],[\overline{\mathcal{J}}])$ by requiring that the total flux $F_{(\mathcal{T}, \mathcal{Y}, \overline{\mathcal{Y}})}$ has vanishing conformal weights; see table 1.

The expression (5.1) can be seen as a pairing $\langle\cdot, \cdot\rangle$ between the BMS generators of the algebra and the momentum fluxes:

$$
\mathfrak{b m s}_{4}^{*} \times \mathfrak{b m s}_{4} \mapsto \mathbb{R}:((\mathcal{P},[\mathcal{J}],[\overline{\mathcal{J}}]),(\mathcal{T}, \mathcal{Y}, \overline{\mathcal{Y}})) \rightarrow F_{(\mathcal{T}, \mathcal{Y}, \overline{\mathcal{Y}})}=\langle(\mathcal{P},[\mathcal{J}],[\overline{\mathcal{J}}]),(\mathcal{T}, \mathcal{Y}, \overline{\mathcal{Y}})\rangle
$$

where $\mathfrak{b m s}_{4}^{*}$ denotes the dual of $\mathfrak{b m s}_{4}$. Indeed, it is linear in both entries and non-degeneracy comes from the fact that we have considered equivalence classes $[\mathcal{J}]$ and $[\overline{\mathcal{J}}]$ of super angular momentum fluxes (4.15). Using this pairing, the transformations laws (4.9) and (4.18) can be interpreted as the coadjoint representation of $\mathfrak{b m s}_{4}[18]$. In particular, it has been shown in that reference that there exists a (pre-)momentum map between the solution space of non-radiative asymptotically flat spacetimes and the dual of the global BMS

\footnotetext{
${ }^{7}$ Notice that to obtain the standard normalization for the BMS fluxes, one should multiply the expression (5.1) by a global factor $-4 i \pi^{2}$ (see also footnote 2 ).
} 
algebra $\mathfrak{s o}(3,1) \forall \mathfrak{s}$. Here, we have extended these results for radiative spacetimes and for extended BMS algebra (Witt $\oplus \overline{\text { Witt }}) \oplus \mathfrak{s}^{*}$ by considering the fluxes on $\mathscr{I}^{+}$, which are $u$-integrated expressions in terms of the solution space, rather than surface charges. These results rely crucially on the falloff conditions (2.20) at the corners of $\mathscr{I}^{+}$and the fact that the BMS fluxes are determined by the values of the surface charges at the corners.

Now, using the basis dual to the one used for the expansion of the generators in (4.1) and (4.3) [18], it is instructive to expand the BMS momentum fluxes as in (3.7):

$$
\begin{aligned}
\mathcal{P}(z, \bar{z}) & =\sum_{k, l} p_{k, l} \mathscr{T}_{*}^{k, l}, & \mathscr{T}_{*}^{k, l} & ={ }_{\frac{3}{2}, \frac{3}{2}} Z_{-l,-k}=z^{-\frac{3}{2}+l} \bar{z}^{-\frac{3}{2}+k}, \\
\mathcal{J}(z, \bar{z}) & =\sum_{m} j_{m} \mathcal{Y}_{*}^{m}, & \mathcal{Y}_{*}^{m} & ={ }_{1,2} Z_{0,-m}=z^{-1} \bar{z}^{-2+m}, \\
\overline{\mathcal{J}}(z, \bar{z}) & =\sum_{m} \bar{j}_{m} \overline{\mathcal{Y}}_{*}^{m}, & \overline{\mathcal{Y}}_{*}^{m} & ={ }_{2,1} Z_{-m, 0}=z^{-2+m} \bar{z}^{-1} .
\end{aligned}
$$

with $\bar{p}_{k, l}=p_{l, k}$ so that $\mathcal{P}$ is real. In terms of the above expansions, the infinitesimal variations (4.9) and (4.18) are encoded in the coadjoint representation of $\mathfrak{b m s}_{4}$, written $\mathrm{ad}^{*}$, as follows [18]:

$$
\begin{array}{rlrl}
\operatorname{ad}_{\mathcal{Y}_{m}}^{*} \mathcal{Y}_{*}^{n} & =(-2 m+n) \mathcal{Y}_{*}^{n-m}, & \operatorname{ad}_{\mathcal{Y}_{m}}^{*} \overline{\mathcal{Y}}_{*}^{n}=(-2 m+n) \overline{\mathcal{Y}}_{*}^{n-m}, \\
\operatorname{ad}_{\mathcal{Y}_{m}}^{*} \mathscr{T}_{*}^{k, l} & =\left(-\frac{3}{2} m+k\right) \mathscr{T}_{*}^{k-m, l}, & \operatorname{ad}_{\overline{\mathcal{Y}}_{m}}^{*} \mathscr{T}_{*}^{k, l}=\left(-\frac{3}{2} m+l\right) \mathscr{T}_{*}^{k, l-} \\
\operatorname{ad}_{\mathscr{T}_{k, l}}^{*} \mathscr{T}_{*}^{r, s} & =\left(\frac{r-3 k}{2}\right) \delta_{l}^{s} \mathcal{Y}_{*}^{r-k}+\left(\frac{s-3 l}{2}\right) \delta_{k}^{r} \overline{\mathcal{Y}}_{*}^{s-l}, & & \\
\operatorname{ad}_{\mathcal{Y}_{m}}^{*} \overline{\mathcal{Y}}_{*}^{n} & =0=\operatorname{ad}_{\overline{\mathcal{Y}}_{m}}^{*} \overline{\mathcal{Y}}_{*}^{n}, & \operatorname{ad}_{\mathscr{T}_{k, l}}^{*} \mathcal{Y}_{*}^{m}=0=\operatorname{ad}_{\mathscr{T}_{k, l}}^{*} \overline{\mathcal{Y}}_{*}^{m} .
\end{array}
$$

Similarly, the soft/hard BMS fluxes

$$
F_{(\mathcal{T}, \mathcal{Y}, \overline{\mathcal{Y}})}^{\text {soft } / \text { hard }}=\int_{\mathcal{S}} \frac{d z d \bar{z}}{(2 i \pi)^{2}}\left[\mathcal{T} \mathcal{P}_{\text {soft } / \text { hard }}+\mathcal{Y} \overline{\mathcal{J}}_{\text {soft } / \text { hard }}+\overline{\mathcal{Y}} \mathcal{J}_{\text {soft } / \text { hard }}\right]
$$

play the role of pairing for the soft/hard sectors. As discussed in section 4, since the soft and hard parts of the momentum fluxes transform separately as (4.9) and (4.18) (see (4.12) and $(4.21)$ ), they also transform in the coadjoint representation of $\mathfrak{b m s}_{4}$ for the appropriate pairing (5.5).

We define the bracket between the BMS fluxes as

$$
\left\{F_{\left(\mathcal{T}_{1}, \mathcal{Y}_{1}, \overline{\mathcal{Y}}_{1}\right)}, F_{\left(\mathcal{T}_{2}, \mathcal{Y}_{2}, \overline{\mathcal{Y}}_{2}\right)}\right\}=\delta_{\left(\mathcal{T}_{1}, \mathcal{Y}_{1}, \overline{\mathcal{Y}}_{1}\right)} F_{\left(\mathcal{T}_{2}, \mathcal{Y}_{2}, \overline{\mathcal{Y}}_{2}\right)}
$$

As shown in [25], the BMS fluxes (5.1) satisfy the algebra

$$
\left\{F_{\left(\mathcal{T}_{1}, \mathcal{Y}_{1}, \overline{\mathcal{Y}}_{1}\right)}, F_{\left(\mathcal{T}_{2}, \mathcal{Y}_{2}, \overline{\mathcal{Y}}_{2}\right)}\right\}=-F_{\left[\left(\mathcal{T}_{1}, \mathcal{Y}_{1}, \overline{\mathcal{Y}}_{1}\right),\left(\mathcal{T}_{2}, \mathcal{Y}_{2}, \overline{\mathcal{Y}}_{2}\right)\right]}
$$

which implies that the bracket (5.6) corresponds to the Kirillov-Kostant Poisson bracket 
on $\mathfrak{b m s}_{4}^{*}$. In terms of the momentum fluxes, the bracket (5.6) can be written explicitly as

$$
\begin{aligned}
& \{\overline{\mathcal{J}}(z, \bar{z}), \overline{\mathcal{J}}(w, \bar{w})\}=\delta^{2}(z-w) \partial_{w} \overline{\mathcal{J}}(w, \bar{w})+2 \partial_{w} \delta^{2}(z-w) \overline{\mathcal{J}}(w, \bar{w}), \\
& \{\overline{\mathcal{J}}(z, \bar{z}), \mathcal{J}(w, \bar{w})\}=\delta^{2}(z-w) \partial_{w} \overline{\mathcal{J}}(w, \bar{w})+\partial_{w} \delta^{2}(z-w) \overline{\mathcal{J}}(w, \bar{w}), \\
& \{\mathcal{P}(z, \bar{z}), \overline{\mathcal{J}}(w, \bar{w})\}=\frac{1}{2} \delta^{2}(z-w) \partial_{w} \mathcal{P}(w, \bar{w})+\frac{3}{2} \partial_{w} \delta^{2}(z-w) \mathcal{P}(w, \bar{w}), \\
& \{\overline{\mathcal{J}}(z, \bar{z}), \mathcal{P}(w, \bar{w})\}=\delta^{2}(z-w) \partial_{w} \mathcal{P}(w, \bar{w})+\frac{3}{2} \partial_{w} \delta^{2}(z-w) \mathcal{P}(w, \bar{w}), \\
& \{\mathcal{P}(z, \bar{z}), \mathcal{P}(w, \bar{w})\}=0
\end{aligned}
$$

together with the complex conjugate relations. In particular, these relations reproduce the desired variations (4.9) and (4.18). Similarly, the flux algebra (5.7) can be written in terms of the momentum fluxes as

$$
\begin{aligned}
& \{\overline{\mathcal{J}}(z, \bar{z}), \overline{\mathcal{J}}(w, \bar{w})\}=-[\overline{\mathcal{J}}(z, \bar{z})+\overline{\mathcal{J}}(w, \bar{w})] \partial_{z} \delta^{2}(z-w) \\
& \{\overline{\mathcal{J}}(z, \bar{z}), \mathcal{J}(w, \bar{w})\}=-\left[\partial_{z} \delta^{2}(z-w) \mathcal{J}(z, \bar{z})-\partial_{\bar{z}} \delta^{2}(z-w) \overline{\mathcal{J}}(w, \bar{w})\right], \\
& \{\mathcal{P}(z, \bar{z}), \overline{\mathcal{J}}(w, \bar{w})\}=-\left[\frac{1}{2} \mathcal{P}(z, \bar{z})+\mathcal{P}(w, \bar{w})\right] \partial_{z} \delta^{2}(z-w), \\
& \{\mathcal{P}(z, \bar{z}), \mathcal{P}(w, \bar{w})\}=0
\end{aligned}
$$

together with the complex conjugate relations.

The bracket that has been considered up to this point is associated with the total BMS flux (5.1). However, as discussed around equation (5.5) above, one could study the soft/hard sectors separately and consider the appropriate induced bracket on each of them. More explicitly, assuming that the soft and hard sectors factorize [50], we have

$$
\left\{F_{\left(\mathcal{T}_{1}, \mathcal{Y}_{1}, \overline{\mathcal{Y}}_{1}\right)}^{\text {soft }}, F_{\left(\mathcal{T}_{2}, \mathcal{Y}_{2}, \overline{\mathcal{Y}}_{2}\right)}^{\text {hard }}\right\}=0, \quad\left\{F_{\left(\mathcal{T}_{1}, \mathcal{Y}_{1}, \overline{\mathcal{Y}}_{1}\right)}^{\text {soft/hard }}, F_{\left(\mathcal{T}_{2}, \mathcal{Y}_{2}, \overline{\mathcal{Y}}_{2}\right)}^{\text {soft } / \text { hard }}\right\}=-F_{\left[\left(\mathcal{T}_{1}, \mathcal{Y}_{1}, \overline{\mathcal{Y}}_{1}\right),\left(\mathcal{T}_{2}, \mathcal{Y}_{2}, \overline{\mathcal{Y}}_{2}\right)\right]}^{\text {soft/hard }},
$$

where the second relation is straightforwardly obtained by using the first of (5.10), the definition of the bracket (5.6) and the results (4.12) and (4.21). Henceforth, (5.8) and (5.9) can be specified to soft/hard sectors separately. In the following, since we want to relate the BMS flux algebra with the CCFT currents, we will focus on the soft sector.

\section{Momentum fluxes and CCFT operators}

One of the starting points of celestial holography was the remarkable observation that Weinberg's leading soft graviton theorem could be reformulated as the Ward identity arising from the insertion of a $\left(\frac{3}{2}, \frac{1}{2}\right)$ Kac-Moody current $P(z, \bar{z})$, called the supertranslation operator $[6,12]$. Similarly, it was later shown that the subleading soft graviton theorem [69] could be rewritten as an insertion of a $(2,0)$ operator $T(z)$, identified as the stress-tensor of the celestial CFT, reproducing the Ward identity of a $2 d$ CFT [13]. Although the supertranslation operator $P(z, \bar{z})$ and the stress-tensor $T(z)$ play a fundamental role in celestial holography, their precise relation to the bulk solution space in presence of superrotations (with the inclusion of $N_{A B}^{v a c}$ for consistency of the phase space) and their transformation 
properties under the extended BMS symmetries have not yet been explicitly worked out. In this section, we explore these aspects and relate the BMS momentum fluxes introduced above with these CCFT operators.

The supertranslation operator $P(z, \bar{z})$ and its complex conjugate $\bar{P}(z, \bar{z})$ of weights $\left(\frac{3}{2}, \frac{1}{2}\right)$ and $\left(\frac{1}{2}, \frac{3}{2}\right)$, respectively, can be related to the soft supermomentum flux $\mathcal{P}_{\text {soft }}(z, \bar{z})$ as

$$
\mathcal{P}_{\text {soft }}(z, \bar{z})=\overline{\mathscr{D}} P(z, \bar{z})+\mathscr{D} \bar{P}(z, \bar{z})
$$

where the derivative operators $\mathscr{D}$ and $\overline{\mathscr{D}}$ are defined in (3.5). From (4.11) and (6.1), one deduces the explicit expression of $P(z, \bar{z})$ and $\bar{P}(z, \bar{z})$ in terms of the bulk metric:

$$
\begin{aligned}
P(z, \bar{z}) & =\overline{\mathscr{D}} \mathscr{N}^{(0)} \\
& =\frac{1}{16 \pi G} \int_{-\infty}^{+\infty} d u\left(\Omega_{S} \bar{\Omega}_{S}\right)^{\frac{1}{2}}\left[\left(D_{\bar{z}}+\frac{1}{2} \bar{\partial} \Phi\right) \hat{N}_{z z}\right] \\
& =\frac{1}{16 \pi G}\left(\Omega_{S} \bar{\Omega}_{S}\right)^{\frac{1}{2}}\left(D_{\bar{z}}+\frac{1}{2} \bar{\partial} \Phi\right)\left[\Delta C N_{z z}^{v a c}-2 D_{z}^{2} \Delta C\right],
\end{aligned}
$$

together with the complex conjugate expression for $\bar{P}(z, \bar{z})$. To obtain the last equality, we used the falloffs (2.20). Comparing with (4.14), one can rewrite (6.1) as

$$
\mathcal{P}_{\text {soft }}(z, \bar{z})=2 \overline{\mathscr{D}} P(z, \bar{z})=2 \mathscr{D} \bar{P}(z, \bar{z})
$$

which is a direct consequence of the electricity condition (2.22). Notice that the expression of the supertranslation operator (6.2) that we are using is compatible with the one initially proposed in $[6,12]$ when setting $N_{A B}^{v a c}=0$. The additional terms that we have allow us to have nicer transformation laws under the action of BMS symmetries. In particular, the supertranslation operator is an actual Virasoro primary rather then a descendent thanks to the use of the derivative operators (3.5). Indeed, an explicit computation gives

$$
\left\{F_{(\mathcal{T}, \mathcal{Y}, \overline{\mathcal{Y}})}^{\text {soft }}, P\right\}=\delta_{(\mathcal{T}, \mathcal{Y}, \overline{\mathcal{Y}})} P=\mathcal{Y} \partial P+\overline{\mathcal{Y}} \bar{\partial} P+\frac{3}{2} \partial \mathcal{Y} P+\frac{1}{2} \bar{\partial} \overline{\mathcal{Y}} P
$$

or, equivalently,

$$
\begin{aligned}
& \left\{\mathcal{P}_{\text {soft }}(z, \bar{z}), P(w, \bar{w})\right\}=0 \\
& \left\{\overline{\mathcal{J}}_{\text {soft }}(z, \bar{z}), P(w, \bar{w})\right\}=\delta^{2}(z-w) \partial_{w} P(w, \bar{w})+\frac{3}{2} \partial_{w} \delta^{2}(z-w) P(w, \bar{w}), \\
& \left\{\mathcal{J}_{\text {soft }}(z, \bar{z}), P(w, \bar{w})\right\}=\delta^{2}(z-w) \partial_{\bar{w}} P(w, \bar{w})+\frac{1}{2} \partial_{\bar{w}} \delta^{2}(z-w) P(w, \bar{w}) .
\end{aligned}
$$

We have the analogous results for $\bar{P}$.

The stress-tensor of the CCFT is encoded in the complex conformal fields $T(z)$ and $\bar{T}(\bar{z})$ of weights $(2,0)$ and $(0,2)$, respectively. We observe that it can be constructed from the super angular momentum flux introduced in section 4 as

$$
T(z)=\oint_{\mathcal{C}} \frac{d \bar{z}}{2 i \pi} \overline{\mathcal{J}}_{\text {soft }}(z, \bar{z}), \quad \bar{T}(\bar{z})=\oint_{\mathcal{C}} \frac{d z}{2 i \pi} \mathcal{J}_{\text {soft }}(z, \bar{z})
$$


Since total derivatives can be dropped out, the definition (6.6) does not depend on the particular representatives of $\mathcal{J}_{\text {soft }}(z, \bar{z})$ and $\overline{\mathcal{J}}_{\text {soft }}(z, \bar{z})$ in the equivalent classes (4.15). The stress-tensor is related to the soft part of the flux for superrotations in (5.5) through

$$
F_{\mathcal{Y}}^{\text {soft }}=\int_{\mathcal{S}} \frac{d z d \bar{z}}{(2 i \pi)^{2}} \mathcal{Y}(z) \overline{\mathcal{J}}_{\text {soft }}(z, \bar{z})=\oint_{\mathcal{C}} \frac{d z}{2 i \pi} \mathcal{Y}(z) T(z)
$$

and the complex conjugate relation for $F_{\overline{\mathcal{Y}}}^{\text {soft }}$. One can recognize the last expression in (6.7) as the soft part of the superrotation charge $[9,13]$. From (4.20) and (6.6), one deduces the explicit expression of $T$ in terms of the bulk metric:

$$
\begin{aligned}
T(z)=-\frac{1}{16 \pi G} \oint_{\mathcal{C}} \frac{d \bar{z}}{2 i \pi} \int_{-\infty}^{+\infty} & d u\left(\Omega_{S} \bar{\Omega}_{S}\right)^{-1}\left[u\left(D_{z}^{3}-2 N_{z z}^{v a c} D_{z}-D_{z} N_{z z}^{v a c}\right) \hat{N}^{z z}\right. \\
& \left.+N^{z z} D_{z}\left[\left(D_{z}^{2}-\frac{1}{2} N_{z z}^{v a c}\right) C_{-}\right]+3 D_{z} N^{z z}\left(D_{z}^{2}-\frac{1}{2} N_{z z}^{v a c}\right) C_{-}\right] .
\end{aligned}
$$

We have the complex conjugate expression for the anti-holomorphic $\bar{T}(\bar{z})$. The relation (6.8) agrees with the one first proposed in [13] when setting $N_{A B}^{v a c}=0=C_{-}$. The decoration with the terms involving the memory fields is turned on once we are considering a vacuum that is not global Minkowski space [27-29] and allows us to have nicer transformation laws. An explicit computation shows that

$$
\left\{F_{(\mathcal{T}, \mathcal{Y}, \overline{\mathcal{Y}})}^{\text {soft }}, T\right\}=\delta_{(\mathcal{T}, \mathcal{Y}, \overline{\mathcal{Y}})} T=\mathcal{Y} \partial T+2 \partial \mathcal{Y} T+\oint_{\mathcal{C}} \frac{d \bar{z}}{2 i \pi}\left[\frac{1}{2} \mathcal{T} \partial \mathcal{P}_{\text {soft }}+\frac{3}{2} \partial \mathcal{T} \mathcal{P}_{\text {soft }}\right]
$$

or, equivalently,

$$
\begin{aligned}
\left\{\mathcal{P}_{\text {soft }}(z, \bar{z}), T(w)\right\} & =\frac{1}{2} \delta(z-w) \partial_{w} \mathcal{P}_{\text {soft }}(w, \bar{z})+\frac{3}{2} \partial_{w} \delta(z-w) \mathcal{P}_{\text {soft }}(w, \bar{z}) \\
\left\{\overline{\mathcal{J}}_{\text {soft }}(z, \bar{z}), T(w)\right\} & =\delta^{2}(z-w) \partial_{w} T(w)+2 \partial_{w} \delta^{2}(z-w) T(w) \\
\left\{\mathcal{J}_{\text {soft }}(z, \bar{z}), T(w)\right\} & =0
\end{aligned}
$$

We again have the analogous results for $\bar{T}$.

Finally, using once more the definitions (6.1) and (6.6), the commutation relations (6.5) and (6.10) lead to

$$
\begin{aligned}
\{P(z, \bar{z}), P(w, \bar{w})\} & =0 \\
\{P(z, \bar{z}), \bar{P}(w, \bar{w})\} & =0, \\
\{T(z), P(w, \bar{w})\} & =\delta(z-w) \partial_{w} P(w, \bar{w})+\frac{3}{2} \partial_{w} \delta(z-w) P(w, \bar{w}), \\
\{\bar{T}(\bar{z}), P(w, \bar{w})\} & =\delta(\bar{z}-\bar{w}) \partial_{\bar{w}} P(w, \bar{w})+\frac{1}{2} \partial_{\bar{w}} \delta(\bar{z}-\bar{w}) P(w, \bar{w}), \\
\{T(z), T(w)\} & =\delta(z-w) \partial_{w} T(w)+2 \partial_{w} \delta(z-w) T(w), \\
\{\bar{T}(\bar{z}), T(w)\} & =0 .
\end{aligned}
$$




\begin{tabular}{|c|c|c|c|c|c|c|c|c|c|c|c|}
\hline$\phi_{h, \bar{h}}$ & $\mathcal{T}$ & $\mathcal{Y}$ & $\mathcal{P}$ & $\mathcal{J}$ & $\mathscr{N}^{(0)}$ & $\mathscr{N}^{(1)}$ & $\mathscr{C}$ & $P$ & $T$ & $\mathscr{D}$ & $d z$ \\
\hline$h$ & $-\frac{1}{2}$ & -1 & $\frac{3}{2}$ & 1 & $\frac{3}{2}$ & 1 & $-\frac{1}{2}$ & $\frac{3}{2}$ & 2 & 1 & -1 \\
\hline $\bar{h}$ & $-\frac{1}{2}$ & 0 & $\frac{3}{2}$ & 2 & $-\frac{1}{2}$ & -1 & $-\frac{1}{2}$ & $\frac{1}{2}$ & 0 & 0 & 0 \\
\hline$J$ & 0 & -1 & 0 & -1 & 2 & 2 & 0 & 1 & 2 & 1 & -1 \\
\hline$\Delta$ & -1 & -1 & 3 & 3 & 1 & 0 & -1 & 2 & 2 & 1 & -1 \\
\hline
\end{tabular}

Table 1. Conformal weights $(h, \bar{h})$ and spin/boost weights $(J, \Delta)$.

The other relations can be obtained by complex conjugation and antisymmetry of the bracket.

The conformal weights $(h, \bar{h})$ and spin/boost weights $(J, \Delta)$ of the relevant objects discussed in this paper are summarized in table 1 (see also [18]). Under complex conjugation, we have $\overline{(h, \bar{h})}=(\bar{h}, h), \overline{(J, \Delta)}=(-J, \Delta)$.

\section{Constraints on CCFT}

Up to this stage, all the results have been obtained from gravitational bulk computations. Some non-local combinations of the solution space have been identified as conformal fields on the celestial Riemann surface whose transformation laws are induced by bulk diffeomorphisms. In this construction, a phase space structure has emerged naturally from the BMS flux algebra. We now study the implications of these results at the quantum level and derive the OPEs between the various conformal operators.

Using standard arguments [56], one can deduce the singular parts of the OPEs between the operators associated with BMS momentum fluxes by starting from their commutation relations (5.8). We have explicitly

$$
\begin{aligned}
\overline{\mathcal{J}}(z, \bar{z}) \overline{\mathcal{J}}(w, \bar{w}) & \sim \frac{2}{(z-w)^{2}(\bar{z}-\bar{w})} \overline{\mathcal{J}}(w, \bar{w})+\frac{1}{(z-w)(\bar{z}-\bar{w})} \partial_{w} \overline{\mathcal{J}}(w, \bar{w}), \\
\overline{\mathcal{J}}(z, \bar{z}) \mathcal{J}(w, \bar{w}) & \sim \frac{1}{(z-w)^{2}(\bar{z}-\bar{w})} \overline{\mathcal{J}}(w, \bar{w})+\frac{1}{(z-w)(\bar{z}-\bar{w})} \partial_{w} \overline{\mathcal{J}}(w, \bar{w}), \\
\mathcal{P}(z, \bar{z}) \overline{\mathcal{J}}(w, \bar{w}) & \sim \frac{3 / 2}{(z-w)^{2}(\bar{z}-\bar{w})} \mathcal{P}(w, \bar{w})+\frac{1 / 2}{(z-w)(\bar{z}-\bar{w})} \partial_{w} \mathcal{P}(w, \bar{w}), \\
\overline{\mathcal{J}}(z, \bar{z}) \mathcal{P}(w, \bar{w}) & \sim \frac{3 / 2}{(z-w)^{2}(\bar{z}-\bar{w})} \mathcal{P}(w, \bar{w})+\frac{1}{(z-w)(\bar{z}-\bar{w})} \partial_{w} \mathcal{P}(w, \bar{w}), \\
\mathcal{P}(z, \bar{z}) \mathcal{P}(w, \bar{w}) & \sim 0
\end{aligned}
$$

together with the complex conjugate relations. The notation " " means equality modulo expressions that are regular as $(z, \bar{z}) \rightarrow(w, \bar{w})$. The third OPE in (7.1) can be deduced from the fourth one using $\mathcal{P}(z, \bar{z}) \overline{\mathcal{J}}(w, \bar{w})=\overline{\mathcal{J}}(w, \bar{w}) \mathcal{P}(z, \bar{z})$. We will avoid writing redundant OPEs in the following.

As a consequence of the factorization between hard and soft sectors in the phase space discussed at the end of section 5, the above OPEs can be written for hard and soft BMS 
momentum fluxes separately. From now on, since we want to find constraints on the CCFT from celestial currents, we will restrict ourselves to the soft sector. The OPEs between soft BMS momentum fluxes and the CCFT operators can be readily deduced from (6.5) and (6.10), leading to:

$$
\begin{aligned}
\mathcal{P}_{\text {soft }}(z, \bar{z}) P(w, \bar{w}) & \sim 0, \\
\overline{\mathcal{J}}_{\text {soft }}(z, \bar{z}) P(w, \bar{w}) & \sim \frac{3 / 2}{(z-w)^{2}(\bar{z}-\bar{w})} P(w, \bar{w})+\frac{1}{(z-w)(\bar{z}-\bar{w})} \partial_{w} P(w, \bar{w}), \\
\mathcal{J}_{\text {soft }}(z, \bar{z}) P(w, \bar{w}) & \sim \frac{1 / 2}{(z-w)(\bar{z}-\bar{w})^{2}} P(w, \bar{w})+\frac{1}{(z-w)(\bar{z}-\bar{w})} \partial_{\bar{w}} P(w, \bar{w}), \\
\mathcal{P}_{\text {soft }}(z, \bar{z}) T(w) & \sim \frac{3 / 2}{(z-w)^{2}} \mathcal{P}_{\text {soft }}(w, \bar{z})+\frac{1 / 2}{(z-w)} \partial_{w} \mathcal{P}_{\text {soft }}(w, \bar{z}), \\
\overline{\mathcal{J}}_{\text {soft }}(z, \bar{z}) T(w) & \sim \frac{2}{(z-w)^{2}(\bar{z}-\bar{w})} T(w)+\frac{1}{(z-w)(\bar{z}-\bar{w})} \partial_{w} T(w), \\
\mathcal{J}_{\text {soft }}(z, \bar{z}) T(w) & \sim 0 .
\end{aligned}
$$

Finally, using the commutation relations (6.11), one obtains the OPEs between CCFT operators:

$$
\begin{aligned}
P(z, \bar{z}) P(w, \bar{w}) & \sim 0, \\
P(z, \bar{z}) \bar{P}(w, \bar{w}) & \sim 0, \\
T(z) P(w, \bar{w}) & \sim \frac{3 / 2}{(z-w)^{2}} P(w, \bar{w})+\frac{1}{(z-w)} \partial_{w} P(w, \bar{w}), \\
\bar{T}(\bar{z}) P(w, \bar{w}) & \sim \frac{1 / 2}{(\bar{z}-\bar{w})^{2}} P(w, \bar{w})+\frac{1}{(\bar{z}-\bar{w})} \partial_{\bar{w}} P(w, \bar{w}), \\
T(z) T(w) & \sim \frac{2}{(z-w)^{2}} T(w)+\frac{1}{(z-w)} \partial_{w} T(w), \\
\bar{T}(\bar{z}) T(w) & \sim 0 .
\end{aligned}
$$

These results are compatible with those given in [57]. They also match with the OPEs found in [10] that were derived from collinear and conformally soft limits of amplitudes, up to the fact that the supertranslation operator that we are considering here is a Virasoro primary rather than a descendant.

Let us now elaborate more on the constraints involving the celestial CFT operators and momentum flux operators with generic conformal operators. To simplify the discussion, we set the memory fields to zero, i.e. $\varphi=0=\bar{\varphi}\left(N_{A B}^{v a c}=0\right)$ and $C_{-}=0$. In celestial holography, a massless particle of energy $\omega$ involved in a scattering process in $4 d$ flat space is associated to an operator $\mathcal{O}(\omega, z, \bar{z})$ (which can depend on other quantum numbers, which are omitted in this notation), where $(z, \bar{z})$ labels the point on the celestial sphere where the particle exits (or enters) spacetime $[11,70]$. Instead of working in the usual momentum basis, a promising celestial dictionary involves the Mellin representation [14-16]

$$
\mathcal{O}_{h, \bar{h}}(z, \bar{z})=\int_{0}^{\infty} d \omega \omega^{\Delta-1} \mathcal{O}(\omega, z, \bar{z}),
$$


which trades the energy $\omega$ for the conformal dimension $\Delta=h+\hbar$. Celestial operators (7.4) indeed enjoy the property of transforming as $2 d$ quasi-primaries. The so-called conformally soft limits [71], for which the conformal dimension takes specific values, lead to $2 d$ currents on the CCFT; see e.g. [71-80].

It was shown that the OPEs involving the components of the CCFT stress-tensor ${ }^{8} T(z)$, $\bar{T}(\bar{z})$ and celestial operators $\mathcal{O}_{h, \bar{h}}$ representing gauge bosons and gravitons are given by

$$
\begin{aligned}
T(z) \mathcal{O}_{h, \bar{h}}(w, \bar{w}) & \sim \frac{h}{(z-w)^{2}} \mathcal{O}_{h, \bar{h}}(w, \bar{w})+\frac{1}{(z-w)} \partial_{w} \mathcal{O}_{h, \bar{h}}(w, \bar{w}), \\
\bar{T}(z) \mathcal{O}_{h, \bar{h}}(w, \bar{w}) & \sim \frac{\bar{h}}{(\bar{z}-\bar{w})^{2}} \mathcal{O}_{h, \bar{h}}(w, \bar{w})+\frac{1}{(\bar{z}-\bar{w})} \partial_{\bar{w}} \mathcal{O}_{h, \bar{h}}(w, \bar{w}),
\end{aligned}
$$

which implies that these operators are Virasoro primaries. These expressions were derived from collinear and conformally soft limits of Einstein-Yang-Mills amplitudes in [10, 81]. We deduce from (7.5) that OPEs involving the super angular momentum flux are of the following form:

$$
\begin{aligned}
\overline{\mathcal{J}}(z, \bar{z}) \mathcal{O}_{h, \bar{h}}(w, \bar{w}) & \sim \frac{h}{(z-w)^{2}(\bar{z}-\bar{w})} \mathcal{O}_{h, \bar{h}}(w, \bar{w})+\frac{1}{(z-w)(\bar{z}-\bar{w})} \partial_{w} \mathcal{O}_{h, \bar{h}}(w, \bar{w}), \\
\mathcal{J}(z, \bar{z}) \mathcal{O}_{h, \bar{h}}(w, \bar{w}) & \sim \frac{\bar{h}}{(z-w)(\bar{z}-\bar{w})^{2}} \mathcal{O}_{h, \bar{h}}(w, \bar{w})+\frac{1}{(z-w)(\bar{z}-\bar{w})} \partial_{\bar{w}} \mathcal{O}_{h, \bar{h}}(w, \bar{w}) .
\end{aligned}
$$

While superrotations lead to the expected expressions (7.5) in a CFT, it is a notorious fact in celestial holography that supertranslation symmetry is more subtle to deal with. It particular, it has been shown that the insertion of the supertranslation operator $P(z, \bar{z})$ into a celestial correlation function gives [10, 71]:

$$
P(z, \bar{z}) \mathcal{O}_{h, \bar{h}}(w, \bar{w}) \sim \frac{1}{(z-w)} \mathcal{O}_{h+\frac{1}{2}, \bar{h}+\frac{1}{2}}(w, \bar{w}) .
$$

This OPE relationship is nothing but the celestial consequence of Weinberg's leading soft graviton theorem, as it can be readily obtained from a Mellin transform of the Ward identity associated to supertranslation symmetry $[6,12]$. As one can see from $(7.7)$, the action of supertranslations (even global ones) leads to a shift of $\left(\frac{1}{2}, \frac{1}{2}\right)$ in the conformal weights of celestial operators. Now one can deduce ${ }^{9}$ the generic form of the OPE between the supermomentum flux operator $\mathcal{P}(z, \bar{z})$ and a celestial operator $\mathcal{O}_{h, \bar{h}}(w, \bar{w})$ :

$$
\mathcal{P}(z, \bar{z}) \mathcal{O}_{h, \bar{h}}(w, \bar{w}) \sim \frac{1}{(z-w)(\bar{z}-\bar{w})} \mathcal{O}_{h+\frac{1}{2}, \bar{h}+\frac{1}{2}}(w, \bar{w}) .
$$

This formula agrees with the expression found in [10], which was obtained by taking successive commutators involving the zero-mode of $P$ and the stress-tensor.

\footnotetext{
${ }^{8}$ This operator is actually obtained from the shadow of the conformally soft operator with $\Delta=0[17,71]$.

${ }^{9}$ To do so, we act on (7.7) with the formal anti-derivative $2 \pi \partial_{\bar{z}}^{-1}=\int d^{2} y \frac{1}{z-y}$ and make use of formulae for $2 d$ conformal integrals as in [82].
} 


\section{Discussion}

We now conclude by discussing some possible extensions and consequences of the results presented in this work.

Surface charges versus fluxes. We have argued that the fluxes are more natural objects from the point of view of the CCFT than the surface charges at fixed value of the retarded time $u$. In particular, the fluxes are completely determined by the values of the surface charges at the corners $\mathscr{I}_{ \pm}^{+}$of null infinity, which are non-radiative regions of the spacetime. The statement of the closure of the flux algebra (5.7) can then be recast as the closure of the surface charge algebra at $\mathscr{I}_{ \pm}^{+}$, without the need of modified bracket or the appearance of 2-cocycle. This echoes with recent works suggesting that symmetries are encoded at the corners of hypersurfaces [66, 83-88]. The price to pay for considering fluxes at $\mathscr{I}^{+}$ instead of surface charges is that we lose information on the local flux-balance laws such as the Bondi mass loss formula. Henceforth, both point of views are complementary: the integrated fluxes describe the state of a system, while the surface charges point of view provides information on its dynamics.

Central charge in the CCFT. In the $\mathrm{AdS}_{3} / \mathrm{CFT}_{2}$ correspondence, the central charge of the boundary $\mathrm{CFT}_{2}$ can be read from the Brown-Henneaux central extension [89]. The latter appears classically by computing the charge algebra of large diffeomorphisms in asymptotically $\mathrm{AdS}_{3}$ spacetimes. One might expect that a similar feature would hold in the present context, namely that the possible $\mathrm{CCFT}_{2}$ central extension appears in the classical bulk computation of the charge algebra. However, as stated in (5.7), the BMS flux algebra closes under the standard Peierls bracket and does not exhibit a central term. This indicates that at least the garden-variety type of central charge of the $\mathrm{CCFT}_{2}$ vanishes, which is in agreement with the results found in [10] from computing the TT OPE (see also (7.3)). Let us notice that there is still an imprint of central extension in the transformation of the Liouville stress-tensor that exhibits a Schwarzian derivative (see equation (2.18)).

From extended to generalized BMS. In this paper, we have considered gravity in asymptotically flat spacetimes with a fixed boundary structure. Allowing for some singular punctures on the celestial Riemann surface enables to include the whole Witt $\oplus \overline{\text { Witt }}$ superrotations in the asymptotic symmetry algebra, leading to the extended BMS algebra $[5,7,8]$. If fluctuations of the transverse boundary metric $q_{A B} d x^{A} d x^{B}$ are permitted on the phase space (but keeping a fixed determinant $\sqrt{q}=\sqrt{q}$ ), one gets instead the generalized BMS algebra Diff $\left(S^{2}\right) \forall \mathfrak{s}$ where the superrotations $\operatorname{Diff}\left(S^{2}\right)$ are smooth diffeomorphisms on the celestial sphere [26, 30-32]. Note that these smooth superrotations can be extended to all diffeomorphisms with isolated singularities, written $\operatorname{Diff}\left(S^{2}\right)^{*}$, if the celestial sphere admits some punctures. The latter singular extension is relevant when considering Ward identities of the S-matrix and the relation with subleading soft graviton theorems $[9,30,31,75]$. In particular, we have Witt $\oplus \overline{\operatorname{Witt}} \subset \operatorname{Diff}\left(S^{2}\right)^{*}$, which implies that a notion of conformal field can still be defined: a conformal field $\phi_{h, \bar{h}}(z, \bar{z})$ transforms as in (3.3) under the subgroup of conformal coordinate transformations accompanied by 
a compensating Weyl rescaling to maintain a fixed determinant. The fluxes of charges associated with generalized BMS take the form

$$
F_{(\mathcal{T}, \mathcal{Y}, \overline{\mathcal{Y}})}^{\text {gen }}=\int_{\mathcal{S}} \frac{d z d \bar{z}}{(2 i \pi)^{2}}[\mathcal{T} \mathcal{P}+\mathcal{Y} \overline{\mathcal{J}}+\overline{\mathcal{Y}} \mathcal{J}]
$$

where the superrotation generators $\mathcal{Y}=\mathcal{Y}(z, \bar{z})$ are now (possibly singular) functions of $(z, \bar{z})$ on the celestial Riemann surface. Consequently, since there is no constrain on $\mathcal{Y}$, one does not need to quotient the super angular momentum fluxes $\mathcal{J}(z, \bar{z})$ and $\mathcal{J}(z, \bar{z})$ by the equivalence relation (4.15). In this generalized BMS case, the precise expressions of $\mathcal{P}, \mathcal{J}$ and $\overline{\mathcal{J}}$ in terms of the solution space and their split into hard/soft sectors can be deduced from the analysis displayed in e.g. [24-26]. They should be such that (4.9), (4.12), (4.18) and (4.21) still hold, so that the results of section 5 concerning the BMS flux algebra remain valid.

As argued in [75], a natural object in this context is the shadow transform ${ }^{10}$ of the stress-tensor, which leads to an operator $\widetilde{T}$ of weights $(-1,1)$ :

$$
\widetilde{T}(w, \bar{w})=\frac{3}{2 \pi} \int d^{2} z \frac{(w-z)^{2}}{(\bar{w}-\bar{z})^{2}} T(z) .
$$

It has the following OPE: ${ }^{11}$

$$
\widetilde{T}(w, \bar{w}) \mathcal{O}_{h, \bar{h}}(z, \bar{z}) \sim \frac{-2 h(w-z)}{(\bar{w}-\bar{z})} \mathcal{O}_{h, \bar{h}}(z, \bar{z})+\frac{(w-z)^{2}}{(\bar{w}-\bar{z})} \partial_{z} \mathcal{O}_{h, \bar{h}}(z, \bar{z}) .
$$

Using the observations relating the shadow stress-tensor with the soft part of the superrotation charge that were made in $[17,75],(8.2)$ can be identified (up to a factor) with $F_{\mathcal{Y}=\frac{(z-w))^{2}}{(\bar{z}-\bar{w})}}^{g e n, \text {. }}$. It would be interesting to explore the shadow supermomenta and super angular momenta, which are operators of weights $\left(-\frac{1}{2},-\frac{1}{2}\right)$ and $(0,-1)$. More generally, it would be enlightening to have a full control of shadow transformations from the bulk gravitational phase space point of view.

From generalized to Weyl BMS. A new enhancement of the generalized BMS symmetries has been found recently by allowing the determinant $\sqrt{q}$ of the boundary metric to fluctuate on the phase space [22]. This asymptotic symmetry algebra contains the Weyl rescaling symmetries discussed in $[5,33,34]$ and has the following semi-direct structure:

$$
\left[\operatorname{Diff}\left(S^{2}\right) \oplus \text { Weyl }\right] \bullet \mathfrak{s} \text {. }
$$

It was shown that the charges and fluxes associated with Weyl rescaling symmetries were non-vanishing, which suggests the presence of an additional term in the flux (8.1). It would be interesting to repeat the analysis of the present paper for this case and use the framework developed in $[18,87]$ to treat conformal coordinate transformations and Weyl rescalings separately.

\footnotetext{
${ }^{10}$ The shadow transform is defined as $\widetilde{\mathcal{O}}_{1-h, 1-\bar{h}}(w, \bar{w})=\int d^{2} z(w-z)^{2 h-2}(\bar{w}-\bar{z})^{2 \bar{h}-2} \mathcal{O}_{h, \bar{h}}(z, \bar{z})[82]$, up to a normalization factor which can be chosen in such a way the shadow operation squares to one.

${ }^{11}$ It can be obtained from shadowing the $T(z)$ OPE, or read from expressions involving the $\Delta \rightarrow 0$ conformally soft operator in refs. [10, 17, 90].
} 


\section{Acknowledgments}

We are grateful to Glenn Barnich, Geoffrey Compère, Adrien Fiorucci, Laurent Freidel, Gaston Giribet, Prahar Mitra and Andy Strominger for useful discussions and comments. L.D. is supported by the European Union's Horizon 2020 research and innovation programme under the Marie Skłodowska-Curie grant agreement No. 746297 and by the Austrian Science Fund (FWF), project P 30822. R.R. is supported by the FWF, project P 32581-N.

Open Access. This article is distributed under the terms of the Creative Commons Attribution License (CC-BY 4.0), which permits any use, distribution and reproduction in any medium, provided the original author(s) and source are credited.

\section{References}

[1] H. Bondi, M.G.J. van der Burg and A.W.K. Metzner, Gravitational waves in general relativity VII. Waves from axisymmetric isolated systems, Proc. Roy. Soc. Lond. A 269 (1962) 21 [INSPIRE].

[2] R.K. Sachs, Gravitational waves in general relativity VIII. Waves in asymptotically flat space-times, Proc. Roy. Soc. Lond. A 270 (1962) 103 [inSPIRE].

[3] R. Sachs, Asymptotic symmetries in gravitational theory, Phys. Rev. 128 (1962) 2851 [INSPIRE].

[4] J. de Boer and S.N. Solodukhin, A Holographic reduction of Minkowski space-time, Nucl. Phys. B 665 (2003) 545 [hep-th/0303006] [INSPIRE].

[5] G. Barnich and C. Troessaert, Aspects of the BMS/CFT correspondence, JHEP 05 (2010) 062 [arXiv: 1001.1541] [INSPIRE].

[6] A. Strominger, On BMS Invariance of Gravitational Scattering, JHEP 07 (2014) 152 [arXiv: 1312.2229] [INSPIRE].

[7] G. Barnich and C. Troessaert, Symmetries of asymptotically flat 4 dimensional spacetimes at null infinity revisited, Phys. Rev. Lett. 105 (2010) 111103 [arXiv:0909.2617] [INSPIRE].

[8] G. Barnich and C. Troessaert, Supertranslations call for superrotations, PoS CNCFG2010 (2010) 010 [arXiv:1102.4632] [INSPIRE].

[9] D. Kapec, V. Lysov, S. Pasterski and A. Strominger, Semiclassical Virasoro symmetry of the quantum gravity $\mathcal{S}$-matrix, JHEP 08 (2014) 058 [arXiv:1406.3312] [INSPIRE].

[10] A. Fotopoulos, S. Stieberger, T.R. Taylor and B. Zhu, Extended BMS Algebra of Celestial CFT, JHEP 03 (2020) 130 [arXiv:1912.10973] [INSPIRE].

[11] A. Strominger, Lectures on the Infrared Structure of Gravity and Gauge Theory, Princeton University Press, (2018) [ISBN: 9780691179506].

[12] T. He, V. Lysov, P. Mitra and A. Strominger, BMS supertranslations and Weinberg's soft graviton theorem, JHEP 05 (2015) 151 [arXiv: 1401.7026] [INSPIRE].

[13] D. Kapec, P. Mitra, A.-M. Raclariu and A. Strominger, 2D Stress Tensor for 4 D Gravity, Phys. Rev. Lett. 119 (2017) 121601 [arXiv: 1609.00282] [InSPIRE]. 
[14] S. Pasterski, S.-H. Shao and A. Strominger, Flat Space Amplitudes and Conformal Symmetry of the Celestial Sphere, Phys. Rev. D 96 (2017) 065026 [arXiv:1701.00049] [InSPIRE].

[15] S. Pasterski, S.-H. Shao and A. Strominger, Gluon Amplitudes as 2d Conformal Correlators, Phys. Rev. D 96 (2017) 085006 [arXiv:1706.03917] [INSPIRE].

[16] S. Pasterski and S.-H. Shao, Conformal basis for flat space amplitudes, Phys. Rev. D 96 (2017) 065022 [arXiv: 1705. 01027] [INSPIRE].

[17] C. Cheung, A. de la Fuente and R. Sundrum, $4 D$ scattering amplitudes and asymptotic symmetries from 2D CFT, JHEP 01 (2017) 112 [arXiv:1609.00732] [INSPIRE].

[18] G. Barnich and R. Ruzziconi, Coadjoint representation of the BMS group on celestial Riemann surfaces, JHEP 06 (2021) 079 [arXiv:2103.11253] [INSPIRE].

[19] R.M. Wald and A. Zoupas, A General definition of 'conserved quantities' in general relativity and other theories of gravity, Phys. Rev. D 61 (2000) 084027 [gr-qc/9911095] [inSPIRE].

[20] G. Barnich and C. Troessaert, BMS charge algebra, JHEP 12 (2011) 105 [arXiv:1106.0213] [INSPIRE].

[21] J. Distler, R. Flauger and B. Horn, Double-soft graviton amplitudes and the extended BMS charge algebra, JHEP 08 (2019) 021 [arXiv:1808.09965] [INSPIRE].

[22] L. Freidel, R. Oliveri, D. Pranzetti and S. Speziale, The Weyl BMS group and Einstein's equations, JHEP 07 (2021) 170 [arXiv:2104.05793] [INSPIRE].

[23] L. Freidel, R. Oliveri, D. Pranzetti and S. Speziale, Extended corner symmetry, charge bracket and Einstein's equations, JHEP 09 (2021) 083 [arXiv:2104.12881] [INSPIRE].

[24] M. Campiglia and J. Peraza, Generalized BMS charge algebra, Phys. Rev. D 101 (2020) 104039 [arXiv: 2002.06691] [INSPIRE].

[25] G. Compère, A. Fiorucci and R. Ruzziconi, The $\Lambda$-BMS 4 charge algebra, JHEP 10 (2020) 205 [arXiv: 2004.10769] [INSPIRE].

[26] G. Compère, A. Fiorucci and R. Ruzziconi, Superboost transitions, refraction memory and super-Lorentz charge algebra, JHEP 11 (2018) 200 [Erratum ibid. 04 (2020) 172] [arXiv: 1810.00377] [INSPIRE].

[27] G. Compère and J. Long, Vacua of the gravitational field, JHEP 07 (2016) 137 [arXiv: 1601.04958] [INSPIRE].

[28] G. Compère and J. Long, Classical static final state of collapse with supertranslation memory, Class. Quant. Grav. 33 (2016) 195001 [arXiv:1602.05197] [INSPIRE].

[29] G. Compère, Bulk supertranslation memories: a concept reshaping the vacua and black holes of general relativity, Int. J. Mod. Phys. D 25 (2016) 1644006 [arXiv:1606.00377] [INSPIRE].

[30] M. Campiglia and A. Laddha, New symmetries for the Gravitational S-matrix, JHEP 04 (2015) 076 [arXiv: 1502.02318] [inSPIRE].

[31] M. Campiglia and A. Laddha, Asymptotic symmetries and subleading soft graviton theorem, Phys. Rev. D 90 (2014) 124028 [arXiv: 1408.2228] [INSPIRE].

[32] E.E. Flanagan, K. Prabhu and I. Shehzad, Extensions of the asymptotic symmetry algebra of general relativity, JHEP 01 (2020) 002 [arXiv:1910.04557] [INSPIRE].

[33] G. Barnich and C. Troessaert, Finite BMS transformations, JHEP 03 (2016) 167 [arXiv: 1601.04090] [INSPIRE]. 
[34] G. Barnich, P. Mao and R. Ruzziconi, BMS current algebra in the context of the Newman-Penrose formalism, Class. Quant. Grav. 37 (2020) 095010 [arXiv:1910.14588] [INSPIRE].

[35] R. Ruzziconi, On the Various Extensions of the BMS Group, Ph.D. thesis, (2020). arXiv:2009.01926 [INSPIRE].

[36] L.A. Tamburino and J.H. Winicour, Gravitational Fields in Finite and Conformal Bondi Frames, Phys. Rev. 150 (1966) 1039 [inSPIRE].

[37] J. Winicour, Logarithmic asymptotic flatness, Found. Phys. 15 (1985) 605.

[38] L. Andersson and P.T. Chrusciel, On 'hyperboloidal' Cauchy data for vacuum Einstein equations and obstructions to smoothness of 'null infinity', Phys. Rev. Lett. 70 (1993) 2829 [gr-qc/9304019] [INSPIRE].

[39] P.T. Chrusciel, M.A.H. MacCallum and D.B. Singleton, Gravitational waves in general relativity: XIV. Bondi expansions and the polyhomogeneity of Scri, gr-qc/9305021 [INSPIRE].

[40] L. Andersson and P. Chrusciel, On 'hyperboloidal' Cauchy data for vacuum Einstein equations and obstructions to smoothness of Scri, Commun. Math. Phys. 161 (1994) 533 [INSPIRE].

[41] A. Ashtekar, J. Bicak and B.G. Schmidt, Behavior of Einstein-Rosen waves at null infinity, Phys. Rev. D 55 (1997) 687 [gr-qc/9608041] [INSPIRE].

[42] H. Friedrich, Peeling or not peeling - is that the question?, Class. Quant. Grav. 35 (2018) 083001 [arXiv: 1709.07709] [INSPIRE].

[43] Y. Angelopoulos, S. Aretakis and D. Gajic, Logarithmic corrections in the asymptotic expansion for the radiation field along null infinity, J. Hyperbol. Diff. Equat. 16 (2019) 1 [arXiv: 1712.09977] [INSPIRE].

[44] J.A.V. Kroon, Conserved quantities for polyhomogeneous space-times, Class. Quant. Grav. 15 (1998) 2479 [gr-qc/9805094] [INSPIRE].

[45] M. Godazgar and G. Long, BMS charges in polyhomogeneous spacetimes, Phys. Rev. D 102 (2020) 064036 [arXiv:2007.15672] [INSPIRE].

[46] A. Schwimmer and S. Theisen, Entanglement Entropy, Trace Anomalies and Holography, Nucl. Phys. B 801 (2008) 1 [arXiv:0802.1017] [InSPIRE].

[47] R. Geroch, Asymptotic Structure of Space-Time, Springer US, Boston, MA, U.S.A. (1977) [DOI].

[48] A. Ashtekar, Geometry and Physics of Null Infinity, arXiv:1409.1800 [INSPIRE].

[49] K. Nguyen and J. Salzer, The effective action of superrotation modes, JHEP 02 (2021) 108 [arXiv: 2008.03321] [INSPIRE].

[50] M. Campiglia and A. Laddha, BMS Algebra, Double Soft Theorems, and All That, arXiv:2106.14717 [INSPIRE].

[51] A. Strominger and A. Zhiboedov, Gravitational Memory, BMS Supertranslations and Soft Theorems, JHEP 01 (2016) 086 [arXiv:1411.5745] [INSPIRE].

[52] E. Newman and R. Penrose, An Approach to gravitational radiation by a method of spin coefficients, J. Math. Phys. 3 (1962) 566 [INSPIRE]. 
[53] E.T. Newman and R. Penrose, Note on the Bondi-Metzner-Sachs group, J. Math. Phys. 7 (1966) 863 [INSPIRE].

[54] A. Held, E.T. Newman and R. Posadas, The Lorentz group and the sphere, J. Math. Phys. 11 (1970) 3145 [INSPIRE].

[55] V. Kac, Vertex algebras for beginners, AMS, University lecture series 10 (1997) [DOI].

[56] M. Schottenloher, A Mathematical Introduction to Conformal Field Theory, 2 ed., Springer-Verlag Berlin Heidelberg, Lect. Notes Phys. (2008) [DOI].

[57] G. Barnich, Centrally extended BMS4 Lie algebroid, JHEP 06 (2017) 007 [arXiv: 1703.08704] [INSPIRE].

[58] G. Compère and D.A. Nichols, Classical and Quantized General-Relativistic Angular Momentum, arXiv:2103.17103 [INSPIRE].

[59] A. Fiorucci, Leaky covariant phase spaces: theory and application to $\Lambda$-BMS symmetry, Ph.D. Thesis (2021).

[60] J. Lee and R.M. Wald, Local symmetries and constraints, J. Math. Phys. 31 (1990) 725 [INSPIRE].

[61] R.M. Wald, Black hole entropy is the Noether charge, Phys. Rev. D 48 (1993) R3427 [gr-qc/9307038] [INSPIRE].

[62] V. Iyer and R.M. Wald, Some properties of Noether charge and a proposal for dynamical black hole entropy, Phys. Rev. D 50 (1994) 846 [gr-qc/9403028] [INSPIRE].

[63] G. Barnich and F. Brandt, Covariant theory of asymptotic symmetries, conservation laws and central charges, Nucl. Phys. B 633 (2002) 3 [hep-th/0111246] [INSPIRE].

[64] E.E. Flanagan and D.A. Nichols, Conserved charges of the extended Bondi-Metzner-Sachs algebra, Phys. Rev. D 95 (2017) 044002 [arXiv:1510.03386] [InSPIRE].

[65] D. Harlow and J.-Q. Wu, Covariant phase space with boundaries, JHEP 10 (2020) 146 [arXiv: 1906.08616] [INSPIRE].

[66] L. Freidel, M. Geiller and D. Pranzetti, Edge modes of gravity. Part I. Corner potentials and charges, JHEP 11 (2020) 026 [arXiv:2006.12527] [INSPIRE].

[67] G. Compère, R. Oliveri and A. Seraj, The Poincaré and BMS flux-balance laws with application to binary systems, JHEP 10 (2020) 116 [arXiv:1912.03164] [INSPIRE].

[68] A. Elhashash and D.A. Nichols, Definitions of angular momentum and super angular momentum in asymptotically flat spacetimes: Properties and applications to compact-binary mergers, Phys. Rev. D 104 (2021) 024020 [arXiv:2101.12228] [InSPIRE].

[69] F. Cachazo and A. Strominger, Evidence for a New Soft Graviton Theorem, arXiv: 1404.4091 [INSPIRE].

[70] T. He, P. Mitra and A. Strominger, 2D Kac-Moody Symmetry of 4 D Yang-Mills Theory, JHEP 10 (2016) 137 [arXiv:1503.02663] [INSPIRE].

[71] L. Donnay, A. Puhm and A. Strominger, Conformally Soft Photons and Gravitons, JHEP 01 (2019) 184 [arXiv: 1810.05219] [INSPIRE].

[72] D. Nandan, A. Schreiber, A. Volovich and M. Zlotnikov, Celestial Amplitudes: Conformal Partial Waves and Soft Limits, JHEP 10 (2019) 018 [arXiv:1904.10940] [INSPIRE]. 
[73] E. Himwich and A. Strominger, Celestial current algebra from Low's subleading soft theorem, Phys. Rev. D 100 (2019) 065001 [arXiv:1901.01622] [INSPIRE].

[74] M. Pate, A.-M. Raclariu, A. Strominger and E.Y. Yuan, Celestial operator products of gluons and gravitons, Rev. Math. Phys. 33 (2021) 2140003 [arXiv:1910.07424] [INSPIRE].

[75] L. Donnay, S. Pasterski and A. Puhm, Asymptotic Symmetries and Celestial CFT, JHEP 09 (2020) 176 [arXiv: 2005.08990] [INSPIRE].

[76] S. Banerjee, S. Ghosh and R. Gonzo, BMS symmetry of celestial OPE, JHEP 04 (2020) 130 [arXiv: 2002.00975] [INSPIRE].

[77] S. Pasterski, A. Puhm and E. Trevisani, Celestial Diamonds: Conformal Multiplets in Celestial CFT, arXiv:2105.03516 [INSPIRE].

[78] A. Guevara, E. Himwich, M. Pate and A. Strominger, Holographic Symmetry Algebras for Gauge Theory and Gravity, arXiv:2103.03961 [INSPIRE].

[79] A. Strominger, w(1+infinity) and the Celestial Sphere, arXiv:2105.14346 [INSPIRE].

[80] E. Himwich, M. Pate and K. Singh, Celestial Operator Product Expansions and $\mathrm{w}_{1+\infty}$ Symmetry for All Spins, arXiv:2108.07763 [INSPIRE].

[81] A. Fotopoulos and T.R. Taylor, Primary Fields in Celestial CFT, JHEP 10 (2019) 167 [arXiv: 1906.10149] [INSPIRE].

[82] H. Osborn, Conformal Blocks for Arbitrary Spins in Two Dimensions, Phys. Lett. B 718 (2012) 169 [arXiv: 1205.1941] [INSPIRE].

[83] W. Donnelly and L. Freidel, Local subsystems in gauge theory and gravity, JHEP 09 (2016) 102 [arXiv: 1601.04744 ] [INSPIRE].

[84] A. Laddha, S.G. Prabhu, S. Raju and P. Shrivastava, The Holographic Nature of Null Infinity, SciPost Phys. 10 (2021) 041 [arXiv: 2002.02448] [INSPIRE].

[85] L. Freidel, M. Geiller and D. Pranzetti, Edge modes of gravity. Part II. Corner metric and Lorentz charges, JHEP 11 (2020) 027 [arXiv: 2007.03563] [INSPIRE].

[86] L. Freidel, M. Geiller and D. Pranzetti, Edge modes of gravity. Part III. Corner simplicity constraints, JHEP 01 (2021) 100 [arXiv:2007.12635] [INSPIRE].

[87] W. Donnelly, L. Freidel, S.F. Moosavian and A.J. Speranza, Gravitational edge modes, coadjoint orbits, and hydrodynamics, JHEP 09 (2021) 008 [arXiv:2012.10367] [INSPIRE].

[88] L. Ciambelli and R.G. Leigh, Isolated surfaces and symmetries of gravity, Phys. Rev. D 104 (2021) 046005 [arXiv: 2104.07643] [INSPIRE].

[89] J.D. Brown and M. Henneaux, Central Charges in the Canonical Realization of Asymptotic Symmetries: An Example from Three-Dimensional Gravity, Commun. Math. Phys. 104 (1986) 207 [INSPIRE].

[90] T. Adamo, L. Mason and A. Sharma, Celestial amplitudes and conformal soft theorems, Class. Quant. Grav. 36 (2019) 205018 [arXiv:1905.09224] [INSPIRE]. 